\title{
On anisotropic polynomial relations for the elasticity tensor
}

\author{
N. Auffray · B. Kolev · M. Petitot
}

Received: date / Accepted: date

\begin{abstract}
In this paper, we explore new conditions for an elasticity tensor to belong to a given symmetry class. Our goal is to propose an alternative approach to the identification problem of the symmetry class, based on polynomial invariants and covariants of the elasticity tensor $\mathbf{C}$, rather than on spectral properties of the Kelvin representation. We compute a set of algebraic relations which describe precisely the orthotropic $\left(\left[\mathbb{D}_{2}\right]\right)$, trigonal $\left(\left[\mathbb{D}_{3}\right]\right)$, tetragonal $\left(\left[\mathbb{D}_{4}\right]\right)$, transverse isotropic $([\mathrm{SO}(2)])$ and cubic $([\mathbb{O}])$ symmetry classes in $\mathbb{H}^{4}$, the higher irreducible component in the decomposition of $\mathbb{E}$ la. We provide a bifurcation diagram which describes how one "travel" in $\mathbb{H}^{4}$ from a given isotropy class to another. Finally, we study the link between these polynomial invariants and those obtained as the coefficients of the characteristic or the Betten polynomials. We show, in particular, that the Betten invariants do not separate the orbits of the elasticity tensors.
\end{abstract}

Keywords Symmetry classes · Invariants · Anisotropy

PACS $46.05 .+\mathrm{b} \cdot 46.25 .-\mathrm{y} \cdot 46.35 .+\mathrm{z}$

Mathematics Subject Classification (2000) 74 B05 $\cdot$ 15A72

\author{
N. Auffray \\ LMSME, Université Paris-Est, Laboratoire Modélisation et Simulation Multi Echelle, MSME UMR 8208 \\ CNRS, 5 bd Descartes, 77454 Marne-la-Vallée, France \\ E-mail: Nicolas.auffray@univ-mlv.fr \\ B. Kolev \\ LATP, CNRS \& Université d'Aix-Marseille, 39 Rue F. Joliot-Curie, 13453 Marseille Cedex 13, France \\ E-mail: kolev@cmi.univ-mrs.fr \\ M. Petitot \\ LIFL, Université des Sciences et Technologies de Lille I, 59655 Villeneuve d'Ascq Cedex, France \\ E-mail: Michel.Petitot@lifl.fr
}




\section{Introduction}

In this paper we consider the problem of identifying symmetry classes of elastic media symmetries governed by Hooke's law, i.e. identifying the symmetry classes of the elasticity tensor. Different definitions of symmetry classes can be found in the literature, in our approach the definition of Forte and Vianello [17] is retained. In this framework $\mathbb{E}$ la, the vector space of elasticity tensors, is divided into eight conjugacy classes [17] which group tensors having conjugate symmetry groups.

The problem we study here is motivated by experimental needs. When a sample is tested, usually both the symmetries of the material and its orientation are unknown [18]. Expressed in a reference frame (in the laboratory), the measured elasticity tensors, generally, does not exhibit evident symmetry properties, therefore how can the symmetry class of a sample be identified ? This problem is invisible for isotropic materials, but becomes more prominent as the anisotropy of the material increases. For a totally anisotropic (triclinic) material, how can we decide whether two sets of components represent the same material?

This question has already been addressed in the literature. The different approaches are usually based on the spectral decomposition of the elasticity tensor $[29,11,10]$. We propose here an alternative approach using polynomial invariants (and covariants) of the elasticity tensor. Instead of resorting on the spectral decomposition of the elasticity tensor, we use the harmonic decomposition, which is a higher-dimensional analogue of the Fourier decomposition. As established in [17], Ela can be decomposed as follow

$$
\mathbb{E} \text { la } \simeq 2 \mathbb{H}^{0} \oplus 2 \mathbb{H}^{2} \oplus \mathbb{H}^{4} .
$$

in which $\mathbb{H}^{k}$ is space of $k$-th order harmonic tensors, the analogue of Fourier modes. It is interesting to note that $\mathbb{H}^{4}$ and $\mathbb{E}$ la have the same symmetry classes.

In this paper, we develop an invariant-based approach to provide necessary and sufficient conditions for a tensor in $\mathbb{H}^{4}$ to belong to a given isotropy class. This procedure applied to the component $D \in \mathbb{H}^{4}$ of an elasticity tensor, gives invariant necessary conditions for an elasticity tensor to belong to a given symmetry class. Under certain hypothesis, that will be investigated in a forthcoming paper, these conditions can further be generically sufficient.

This approach, investigated in the present contribution, follows a long series of papers $[24,25,8]$. It is interesting to notice that some of the materials introduced here are well-known by the high energy physics community $[1,2,30]$, and has stimulated mathematical researches $[31,27,28]$. However, these methods do not seem to have yet been applied in elasticity.

The objectives of this paper are twofold:

1. Introduce a rigorous (and general) geometric framework to describe anisotropic features of tensor spaces;

2. Parameterize the symmetry classes of $\mathbb{H}^{4}$.

The paper is organized as follows. We begin by recalling the harmonic decomposition of $\mathbb{E}$ la. In section 3 , we introduce the geometric framework used for the analysis of a tensor representation, and in section 4 the geometric parametrization 
of orbit spaces. Although materials introduced in this section are not new, and are probably well-known by most mathematicians and the high-energy physics community, it does not seem to have been yet exploited by the mechanical community. We clarify the geometry behind the so called "normal forms" of elasticity tensors (linear slices) and their ambiguity (monodromy groups). This permits us to justify, on a rigorous mathematical basis, the results given in table 1 and in table 2. Calculations of the monodromy groups for closed subgroups of $\mathrm{SO}(3)$ are given in appendix A. The materials in these sections are rather general and, as the methodology can be applied in other situations, we believe that it was important to properly introduce this framework. Once introduced, these notions are applied to the space $\mathbb{H}^{4}$, in order to provide necessary and sufficient invariant conditions for a tensor in $\mathbb{H}^{4}$ to belong to the following symmetry classes : orthotropic $\left(\left[\mathbb{D}_{2}\right]\right)$, trigonal $\left(\left[\mathbb{D}_{3}\right]\right)$, tetragonal $\left(\left[\mathbb{D}_{4}\right]\right)$, transverse isotropic $([\mathrm{SO}(2)])$ and cubic $([\mathbb{O}])$. For each of these classes, we provide a parametrization of the corresponding stratum by rational expressions involving up to six polynomial invariants. Bifurcation relations between related classes are also computed and summed-up in a diagram (c.f. figure 2 in section 5.6). Finally, in section 6, we prove, as a collateral observation, that the invariants defined by the coefficients of the Betten polynomial [7] do not separate the orbits of Ela. This paper is concluded in section 7 where extension of the method to a broader class of situations is discussed.

\section{Decomposition of the elasticity tensor}

In the theory of linear elasticity, the stress tensor $\sigma$ and the strain tensor $\varepsilon$ are related, at a fixed temperature, by the Hooke's law

$$
\sigma^{i j}=C^{i j k l} \varepsilon_{k l}
$$

The stress tensor $\sigma$ belongs to $S_{2}\left(\mathbb{R}^{3}\right)$, the vector space of contravariant symmetric tensors on $\mathbb{R}^{3}$, while $\varepsilon$ belongs to $S^{2}\left(\mathbb{R}^{3}\right)$, the vector space of covariant symmetric tensors on $\mathbb{R}^{3}$ (both of dimension 6). As a consequence, the elasticity tensor is endowed with minor symmetries

$$
C^{i j k l}=C^{j i k l}=C^{i j l k}
$$

For hyperelastic materials, the stress-strain relation is furthermore assumed to derive from an elastic potential and we must add the major symmetry

$$
C^{i j k l}=C^{k l i j}
$$

The space of (hyper) elasticity tensors is therefore the $21 \mathrm{D}$ vector space

$$
\mathbb{E l a}=S_{2} S_{2}\left(\mathbb{R}^{3}\right)
$$


Remark 2.1. An elasticity tensor $\mathbf{C}$ can also be viewed as a second-rank symmetric tensor $\underline{\mathbf{C}}$ in $S^{2}\left(\mathbb{R}^{3}\right) \simeq \mathbb{R}^{6}$. The associate matrix representation [15] is given by

$$
\underline{\mathbf{C}}=\left(\begin{array}{cccccc}
c_{11} & c_{12} & c_{13} & \sqrt{2} c_{14} & \sqrt{2} c_{15} & \sqrt{2} c_{16} \\
c_{12} & c_{22} & c_{23} & \sqrt{2} c_{24} & \sqrt{2} c_{25} & \sqrt{2} c_{26} \\
c_{13} & c_{23} & c_{33} & \sqrt{2} c_{34} & \sqrt{2} c_{35} & \sqrt{2} c_{36} \\
\sqrt{2} c_{14} & \sqrt{2} c_{24} & \sqrt{2} c_{34} & 2 c_{44} & 2 c_{45} & 2 c_{46} \\
\sqrt{2} c_{15} & \sqrt{2} c_{25} & \sqrt{2} c_{35} & 2 c_{45} & 2 c_{55} & 2 c_{56} \\
\sqrt{2} c_{16} & \sqrt{2} c_{26} & \sqrt{2} c_{36} & 2 c_{46} & 2 c_{56} & 2 c_{66}
\end{array}\right),
$$

where $c_{m n}$ are the components of the elasticity tensor in an orthonormal frame. We use the conventional rule to recode a pair of indices $(i, j)(i, j=1,2,3)$ by a integer $m=\alpha(i, j)(m=1,2, \ldots 6)$, where $\alpha(i, i):=i$ for $1 \leq i \leq 3$ and $\alpha(i, j):=9-(i+j)$ for $i \neq j$.

To each hyperelastic material corresponds an elasticity tensor $\mathbf{C}$ but this association is not unique, it depends of the choice of a fixed orientation of the material. Hence, there is a gauge group. As the material is rotated by an element $\mathbf{g}=\left(g_{i}^{j}\right)$ in the rotation group $\mathrm{SO}(3)$, the elasticity tensor $\mathbf{C}=\left(C^{i j k l}\right)$ is submitted to the following transformation

$$
C^{i j k l} \mapsto g_{p}^{i} g_{q}^{j} g_{r}^{k} g_{s}^{l} C^{p q r s} .
$$

From the point of view of linear elasticity, the classification of elastic materials can be assimilated to the description of the orbits of this $\mathrm{SO}(3)$-representation on the space of elasticity tensors $\mathbb{E}$ la. Since throughout this paper $\mathbb{R}^{3}$ is endowed with its traditional euclidean metric, no distinction will be made between covariant and contravariant components. As a consequence, since now, only lower subscripts will be considered.

This representation can be decomposed into irreducible pieces, known as harmonic tensors (higher dimensional analogue of Fourier modes), and denoted by $\mathbb{H}^{n}\left(\mathbb{R}^{3}\right)$ (or simply by $\mathbb{H}^{n}$ if there is no ambiguity). Each $\mathbb{H}^{n}$ is the space of totally symmetric, traceless tensors of order $n$. It is a vector space of dimension $2 n+1$. This decomposition, known as the harmonic decomposition is the following for $\mathbb{E}$ la

$$
\mathbb{E} \text { la } \simeq 2 \mathbb{H}^{0} \oplus 2 \mathbb{H}^{2} \oplus \mathbb{H}^{4}
$$

It has already been used in the study of anisotropic elasticity tensors [5, 6, 17] and we refer to these references for a deeper insight into this topic.

Each $\mathbf{C} \in \mathbb{E}$ la can therefore be written as $\mathbf{C}=(\lambda, \mu, a, b, D)$ where $\lambda, \mu \in \mathbb{H}^{0}$, $a, b \in \mathbb{H}^{2}$ and $D \in \mathbb{H}^{4}$. The explicit harmonic decomposition is well-known. Given an orthonormal frame $\left(e_{1}, e_{2}, e_{3}\right)$ of the Euclidean space, we get [8]

$$
\begin{aligned}
C_{i j k l}= & \lambda \delta_{i j} \delta_{k l}+\mu\left(\delta_{i k} \delta_{j l}+\delta_{i l} \delta_{j k}\right) \\
& +\delta_{i j} a_{k l}+\delta_{k l} a_{i j} \\
& +\delta_{i k} b_{j l}+\delta_{j l} b_{i k}+\delta_{i l} b_{j k}+\delta_{j k} b_{i l} \\
& +D_{i j k l},
\end{aligned}
$$


where $\left(\delta_{i j}\right)$ are the components of the Euclidean metric $\mathbf{q}$ in the orthonormal frame $\left(e_{i}\right)$. This formula can be inverted to obtain the 5 harmonic components $(\lambda, \mu, a, b, D)$ of C. On $\mathbb{E}$ la $=S^{2} S^{2}\left(\mathbb{R}^{3}\right)$, there are only two different contractions ${ }^{1}$ (or traces)

$$
d_{i j}=\left(\operatorname{tr}_{12} \mathbf{C}\right)_{i j}:=\sum_{k=1}^{3} C_{k k i j}, \quad v_{i j}=\left(\operatorname{tr}_{13} \mathbf{C}\right)_{i j}:=\sum_{k=1}^{3} C_{k i k j}
$$

where $\mathbf{d}$ and $\mathbf{v}$ are known respectively, as the dilatation tensor and the Voigt tensor [14]. Starting with (3) we get

$$
\mathbf{d}=(3 \lambda+2 \mu) \mathbf{q}+3 a+4 b, \quad \mathbf{v}=(\lambda+4 \mu) \mathbf{q}+2 a+5 b .
$$

Taking the traces of each equation, one obtains

$$
\operatorname{tr}(\mathbf{d})=9 \lambda+6 \mu, \quad \operatorname{tr}(\mathbf{v})=3 \lambda+12 \mu,
$$

and, finally:

$$
\begin{aligned}
\lambda & =\frac{1}{15}(2 \operatorname{tr}(\mathbf{d})-\operatorname{tr}(\mathbf{v})), & \mu & =\frac{1}{30}(-\operatorname{tr}(\mathbf{d})+3 \operatorname{tr}(\mathbf{v})), \\
a & =\frac{1}{7}(5 \operatorname{dev}(\mathbf{d})-4 \operatorname{dev}(\mathbf{v})), & b & =\frac{1}{7}(-2 \operatorname{dev}(\mathbf{d})+3 \operatorname{dev}(\mathbf{v})),
\end{aligned}
$$

where $\operatorname{dev}(\mathbf{a}):=\mathbf{a}-\frac{1}{3} \operatorname{tr}(\mathbf{a}) \mathbf{q}$ is the deviatoric part (or traceless part) of the 2 nd-order tensor a.

\section{Geometry of orbit spaces}

In this section, we setup the geometric framework which is required to study rigorously a linear group action on a vector space. Our starting point is a linear representation

$$
\rho: G \rightarrow \mathrm{GL}(V)
$$

of a real, compact Lie group $G$ on a finite dimensional $\mathbb{R}$-vector space $V$. The action on $V$ will be denoted by

$$
g \cdot v:=\rho(g) v, \quad g \in G, v \in V .
$$

3.1 Orbits, symmetry groups and fixed point sets

The set of all vectors $v \in V$ which are related to $v$ by an element $g \in G$ is called the $G$-orbit of $v$ and is denoted by

$$
G \cdot v:=\{g \cdot v \mid g \in G\} .
$$

The $G$-orbits are compact submanifolds of $V[2,12]$.

\footnotetext{
1 The notation $\operatorname{tr}_{i j}$ indicates that the contraction should be done on the $i$-th and $j$-th indices.
} 
The set of transformations of $G$ which leave a given vector $v$ fixed is a closed subgroup of $G$, which is called the isotropy subgroup (or symmetry group) of $v$. It will be denoted by

$$
G_{v}:=\{g \in G ; g \cdot v=v\} .
$$

The symmetry groups of vectors in a same orbit are conjugate

$$
G_{g \cdot v}=g G_{v} g^{-1}, \quad v \in V, g \in G .
$$

Let $H$ be any subgroup of $G$. The set of vectors $v \in V$ which are fixed by $H$

$$
V^{H}:=\{v \in V \mid h . v=v \text { for all } h \in H\},
$$

is called the fixed point set of $H$. It it is a linear subspace of $V$. Notice that, for each $v \in V^{H}, H \subset G_{v}$ and that if $H_{2}=g H_{1} g^{-1}$ then $V^{H_{2}}=g \cdot V^{H_{1}}$. If $H_{1} \subset H_{2}$ then $V^{H_{2}} \subset V^{H_{1}}$. However, it may happen that $V^{H_{2}}=V^{H_{1}}$ but $H_{1} \neq H_{2}$.

Given a subgroup $H$ of $G$, the normalizer of $H$

$$
N(H):=\left\{g \in G \mid g H g^{-1}=H\right\}
$$

is the maximal subgroup of $G$, in which $H$ is a normal subgroup. It can also be defined as the isotropy group of $H$ for the action of $G$ on the set of its subgroup (by conjugacy). The proof of the following important lemma can be found in [19].

Lemma 3.1. For each subgroup $H$ of $G$, the fixed point set $V^{H}$ is $N(H)$-invariant. If $H$ is moreover the isotropy subgroup of some vector $v \in V$, then $N(H)$ is the maximal subgroup of $G$ which leaves invariant $V^{H}$.

\subsection{Symmetry classes and strata}

Two vectors $v_{1}$ and $v_{2}$ are in the same isotropy class (or symmetry class) if their isotropy subgroups are conjugate in $G$, that is if there exists $g \in G$ such that

$$
G_{v_{2}}=g G_{v_{1}} g^{-1}
$$

Due to (5), two vectors $v_{1}$ and $v_{2}$ which are in the same $G$-orbit are in the same isotropy class but the converse is generally false.

The conjugacy class of a subgroup $H$ (i.e. the subset of $\mathscr{P}(G)$ of all subgroups of $G$ which are conjugate to $H$ ) will be denoted by $[H]$. The conjugacy class of an isotropy subgroup will be called an isotropy class. In general, a compact group $G$ has an infinite number of different conjugacy classes, but for a given action, there is only a finite number of different isotropy classes (see [12]). In the particular case of $\mathrm{SO}$ (3)-representation on tensors, this result is known to the mechanical community as a consequence of Hermann's theorem [20,3].

The set of all vectors $v \in V$ in the same isotropy class defined by $[H]$ is denoted $\Sigma_{[H]}$ and called a stratum. It is the union of all $G$-orbits of points which isotropy groups are conjugate to $H$ (it is not a vector subspace in general). There is only a finite 
number of (non empty) strata and each of them is a ( $G$-invariant) smooth submanifold of $V[2,12]$. The partition

$$
V=\Sigma_{\left[H_{0}\right]} \cup \Sigma_{\left[H_{1}\right]} \cup \cdots \cup \Sigma_{\left[H_{n}\right]}
$$

is called the isotropy stratification of $(V, \rho)$.

On the set of conjugacy classes of closed subgroups of a compact group $G$, there is a partial order induced by inclusion. It is defined as follows:

$$
\left[H_{1}\right] \preceq\left[H_{2}\right] \text { iff } H_{1} \text { is conjugate to a subgroup of } H_{2} \text {. }
$$

Endowed with this partial order, the set of isotropy classes ${ }^{2}$ has a least element and a greatest element. This partial order induces a (reverse) partial order relation on strata

$$
\Sigma_{\left[H_{2}\right]} \preceq \Sigma_{\left[H_{1}\right]} \quad \text { iff } \quad\left[H_{1}\right] \preceq\left[H_{2}\right] .
$$

The set of strata inherits therefore the structure of a finite poset (partially ordered set). The topological closure of a stratum $\Sigma_{[H]}$, denoted by $\bar{\Sigma}_{[H]}$, and called shortly the closed stratum associated to $[H]$, corresponds to vectors $v \in V$ such that $v$ has at least isotropy $[H]$. Notice that $\Sigma_{[H]}$ corresponds to vectors $v \in V$ such that $v$ has exactly the isotropy $[H]$. A "closed stratum" is therefore a union of strata. A stratum $\Sigma_{\left[H_{2}\right]} \preceq \Sigma_{\left[H_{1}\right]}$ means that $\Sigma_{\left[H_{2}\right]}$ is contained in the boundary of $\Sigma_{\left[H_{1}\right]}$. A stratum $\Sigma_{\left[H_{2}\right]}$ is said to be adjacent to the stratum $\Sigma_{\left[H_{1}\right]}$ if $\Sigma_{\left[H_{2}\right]} \preceq \Sigma_{\left[H_{1}\right]}$ and there is no other stratum $\Sigma_{[H]}$ between $\Sigma_{\left[H_{2}\right]}$ and $\Sigma_{\left[H_{1}\right]}$ (i.e. $\Sigma_{\left[H_{2}\right]} \preceq \Sigma_{[H]} \preceq \Sigma_{\left[H_{1}\right]}$ ).

Remark 3.2. A $G$-orbit is said to be generic if it belongs to the least isotropy class. The generic stratum $\Sigma_{\left[H_{0}\right]}$, which is the union of generic orbits can be shown to be a dense and open set in $V$. Moreover, for each other stratum $\Sigma_{[H]}$, we have

$$
\operatorname{dim} \Sigma_{[H]}<\operatorname{dim} \Sigma_{\left[H_{0}\right]}
$$

On the opposite side, the stratum which corresponds to the greatest isotropy subgroup is called the minimal stratum ${ }^{3}$.

\subsection{Normal forms and monodromy groups}

It is important to notice that a vector $v \in V$ belongs to the closed strata $\bar{\Sigma}_{[H]}$ (i.e. has at least isotropy $[H]$ ), iff its $G$-orbit intersects the fixed point set $V^{H}$. This observation leads to the possibility to define a normal form ${ }^{4}$ for each $G$-orbit, or at least to reduce the complexity of the problem of describing $G$-orbits.

\footnotetext{
2 The partially ordered set of conjugacy classes of all closed subgroups of $\mathrm{SO}(3)$ is described in section A.

3 Since the isotropy group of the null-vector is $G$ itself, this minimal stratum is always represented by the isotropy class $[G]=\{G\}$. The stratum $\Sigma_{[G]}$ is always reduced to $\{0\}$ if $\rho$ is a non-trivial irreducible representation but it may not be otherwise.

4 In elasticity this is known as the possibility to choose a "natural coordinate system" in which the matrix representation of a given elasticity tensor $\mathbf{C}$ has a lot of zeros.
} 
Example 3.3. For the standard action of the rotation group $\mathrm{SO}(3)$ on the space of $3 \times 3$ symmetric matrices, the least isotropy class is $\left[\mathbb{D}_{2}\right]$. In particular, each symmetric matrix has at least this symmetry. Therefore, the $\mathrm{SO}(3)$-orbit of each matrix meets the fixed point set $V^{\mathbb{D}_{2}}$ which corresponds to diagonal matrices. This gives a (global) normal form representative for each orbit.

This general reduction procedure can be described as a consequence of lemma 3.1. For each subgroup $H$, the linear representation $\rho: G \rightarrow \mathrm{GL}(V)$ induces a linear representation of $N(H)$ on $V^{H}$, obtained by the restriction

$$
\rho_{/ N(H)}: N(H) \longrightarrow \mathrm{GL}\left(V^{H}\right) .
$$

This induced representation is not faithful ${ }^{5}$ because its kernel contains $H$. However, when $H$ is an isotropy group, its kernel is exactly $H$ and we get a faithful linear representation

$$
\rho_{\Gamma^{H}}: \Gamma^{H} \longrightarrow \mathrm{GL}\left(V^{H}\right) \text { where } \Gamma^{H}:=N(H) / H .
$$

Furthermore, two vectors $v_{1}, v_{2}$ in $V^{H} \cap \Sigma_{[H]}$ are in the same $G$-orbit iff there are in the same $N(H)$-orbit. We have therefore reduced (locally, for orbits in $\Sigma_{[H]}$ ) the problem of describing the orbit space $V / G$ to the the orbit space $V^{H} / \Gamma^{H}$. This is especially meaningful when the group $\Gamma^{H}$ is finite, in which case, we say that $V^{H}$ is a linear slice. Then, each $G$-orbit intersects $V^{H}$ at most in a finite number of points and $V^{H} / \Gamma^{H}$ is an orbifold ${ }^{6}$. The cardinal of $\Gamma^{H}$ is equal to the index $[N(H), H]$ of $H$ in its normalizer $N(H)$. It is equal to the number of times, the $G$-orbit of a point in $\Sigma_{[H]}$ intersects the fixed point set $V^{H}$. Each of this point defines a normal form of the orbit (like the diagonal form of a symmetric matrix). But this normal form is not unique in general, these different normal forms are permuted by the group $\Gamma^{H}$ which is called the monodromy group of $V^{H}$ and described this ambiguity (in the case of symmetric matrices this monodromy group corresponds to the permutations of the eigenvalues on the diagonal). It is quite remarkable that this ambiguity (the monodromy group) can be computed a priori, for each isotopy class (see Appendix A).

\subsection{Strata dimensions}

The main formula, which is the basis for all other equations relating dimensions of strata, fixed-point sets and isotropy subgroups is a consequence of the reduction procedure explained in section 3.3. It is summarized by the following lemma.

Lemma 3.4.

$$
\operatorname{dim} \Sigma_{[H]}=\operatorname{dim} V^{H}+\operatorname{dim} G-\operatorname{dim} N(H)
$$

\footnotetext{
5 A representation $\rho: G \rightarrow \mathrm{GL}(V)$ is faithful if the only element $g \in G$ such that $\rho(g)$ is the identity in $\mathrm{GL}(V)$ is the unit element of $G$.

6 Orbifolds have been introduced by Thurston [33]. They generalize manifolds to admit quotients of manifolds by finite groups.
} 
Proof. The main observation is that $\Sigma_{[H]} / G$ is a differentiable manifold, contrary to the whole the orbit space $V / G$ which is not in general. This can be justified as follows (see [12] for a more rigourous proof). Each $G$-orbit in $\Sigma_{[H]}$ meets $V^{H}$, and two vectors in $V^{H} \cap \Sigma_{[H]}$ are in the same $G$-orbit iff they are in the same $\Gamma^{H}$-orbit. Therefore, the orbit spaces $\Sigma_{[H]} / G$ and $\left(V^{H} \cap \Sigma_{[H]}\right) / \Gamma^{H}$ are the same. Notice that the set $V^{H} \cap \Sigma_{[H]}$ is the subspace of vectors in $V^{H}$ whose isotropy classes are exactly $[H]$ (generic elements in $V^{H}$ ). It is an open and dense set in $V^{H}$. But $\Gamma^{H}$ has no fixed point on $V^{H} \cap \Sigma_{[H]}$, from which it can be deduced that $\left(V^{H} \cap \Sigma_{[H]}\right) / \Gamma^{H}$ is a manifold of dimension

$$
\operatorname{dim}\left(V^{H} \cap \Sigma_{[H]}\right) / \Gamma^{H}=\operatorname{dim} V^{H}-\operatorname{dim} \Gamma^{H} .
$$

Moreover, the restriction of the projection map $\pi: V \rightarrow V / G$ to the stratum $\Sigma_{[H]}$

$$
\pi_{/ \Sigma_{[H]}}: \Sigma_{[H]} \rightarrow \Sigma_{[H]} / G
$$

can be shown to be a fiber bundle with fiber $G / H$, i.e. a space that locally looks like $\Sigma_{[H]} / G \times G / H$, and therefore

$$
\operatorname{dim} \Sigma_{[H]}=\operatorname{dim} G / H+\operatorname{dim} \Sigma_{[H]} / G
$$

where

$$
\operatorname{dim} G / H=\operatorname{dim} G-\operatorname{dim} H,
$$

and

$$
\operatorname{dim} \Sigma_{[H]} / G=\operatorname{dim} V^{H}-\operatorname{dim} \Gamma^{H}=\operatorname{dim} V^{H}-(\operatorname{dim} N(H)-\operatorname{dim} H) .
$$

Finally, we get

$$
\operatorname{dim} \Sigma_{[H]}=\operatorname{dim} V^{H}+\operatorname{dim} G-\operatorname{dim} N(H) .
$$

Remark 3.5. The dimension of $V^{H}$ can be computed using the trace formula for the Reynolds operator (see [19]). Letting $\chi_{\rho}$ be the character of the representation $(V, \rho)$, we have

$$
\operatorname{dim} V^{H}=\frac{1}{|H|} \sum_{h \in H} \chi_{\rho}(h),
$$

if $H$ is a finite group (and the preceding formula has to be replaced by the Haar integral over $H$ for an infinite compact Lie group). Explicit analytical formulas based on (7) were obtained in [19] and [4]. 


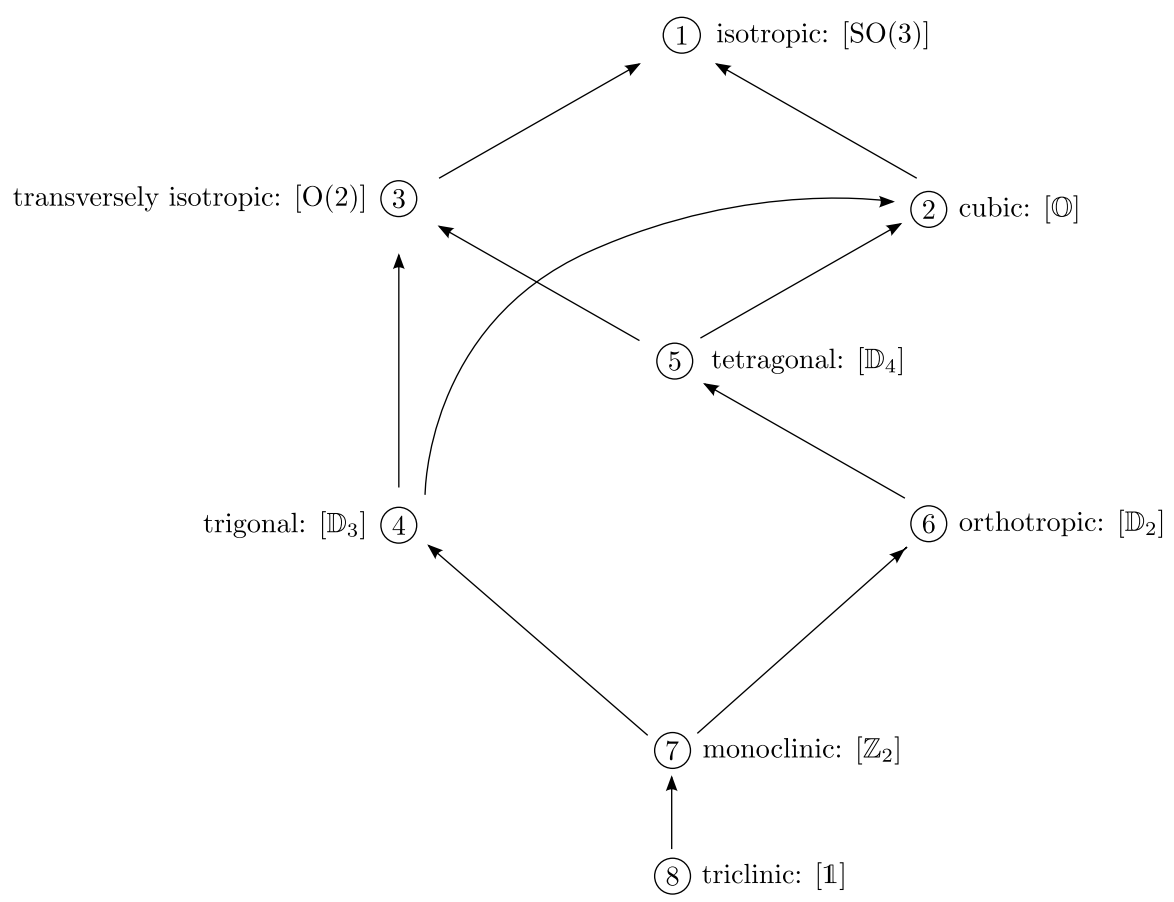

Fig. 1 Adjacency diagram for isotropy classes of Ela.

\subsection{Application to the elasticity tensor}

The isotropy classes for the elasticity tensor have been computed in [17,9] (a general algorithm to compute all the isotropy classes of any $\mathrm{SO}(3)$-representation has been proposed in [23]). There are exactly eight classes: the isotropic class $[\mathrm{SO}(3)]$, the cubic class $[\mathbb{O}]$, the transversely isotropic class $[\mathrm{O}(2)]$, the tetragonal class $\left[\mathbb{D}_{4}\right]$, the trigonal class $\left[\mathbb{D}_{3}\right]$, the orthotropic class $\left[\mathbb{D}_{2}\right]$, the monoclinic class $\left[\mathbb{Z}_{2}\right]$ and the the triclinic class [1] (see Appendix A for the definitions of these classes). The corresponding adjacency diagram is illustrated in figure 1 .

The geometric characterization of $\mathbb{E}$ la and its stratification are summed-up in table 1 , where $\mathfrak{S}_{n}$ is the symmetric group (the group of permutations acting on $n$ elements) and the notations used for the closed subgroup of $\mathrm{SO}(3)$ are given in Appendix A. The results appearing in table 1 are well-known in elasticity, but their constructions are usually ad-hoc and do not stand on rigorous mathematical foundations. The material provided in this section was primary exposed to fix and clarify the geometric framework underneeth ${ }^{7}$. The following remarks should be of importance to relate this framework with popular and historical considerations.

Remark 3.6. The space $V^{H}$ is defined for any closed subgroup $H$, not only for an isotropy subgroup. In elasticity, for instance, fixed-point sets have been considered

\footnotetext{
7 The dimensions of $\Sigma_{[H]}$ and $\Sigma_{[H]} / G$ are obtained using formula (6) of section 3.3, $\operatorname{dim} V^{H}$ is computed using the trace formula (7) and the explicit formulas provided [4].
} 
Table 1 Isotropy classes for $\mathbb{E}$ la.

\begin{tabular}{|l|c|c|c|c|c|c|c|}
\hline Isotropy class & $H$ & $N(H)$ & $\Gamma^{H}$ & $\operatorname{card} \Gamma^{H}$ & $\operatorname{dim} V^{H}$ & $\operatorname{dim} \Sigma_{[H]} / G$ & $\operatorname{dim} \Sigma_{[H]}$ \\
\hline Triclinic & $\mathbb{1}$ & $\mathrm{SO}(3)$ & $\mathrm{SO}(3)$ & $\infty$ & 21 & 18 & 21 \\
Monoclinic & $\mathbb{Z}_{2}$ & $\mathrm{O}(2)$ & $\mathrm{O}(2)$ & $\infty$ & 13 & 12 & 15 \\
Orthotropic & $\mathbb{D}_{2}$ & $\mathbb{O}$ & $\mathfrak{S}_{3}$ & 6 & 9 & 9 & 12 \\
Trigonal & $\mathbb{D}_{3}$ & $\mathbb{D}_{6}$ & $\mathfrak{S}_{2}$ & 2 & 6 & 6 & 9 \\
Tetragonal & $\mathbb{D}_{4}$ & $\mathbb{D}_{8}$ & $\mathfrak{S}_{2}$ & 2 & 6 & 6 & 9 \\
Transverse isotropic & $\mathrm{O}(2)$ & $\mathrm{O}(2)$ & $\mathbb{1}$ & 1 & 5 & 5 & 7 \\
Cubic & $\mathbb{O}$ & $\mathbb{O}$ & $\mathbb{1}$ & 1 & 3 & 3 & 6 \\
Isotropic & $\mathrm{SO}(3)$ & $\mathrm{SO}(3)$ & $\mathbb{1}$ & 1 & 2 & 2 & 2 \\
\hline
\end{tabular}

Table 2 Isotropy classes for $\mathbb{H}^{4}$.

\begin{tabular}{|l|c|c|c|c|c|c|c|}
\hline Isotropy class & $H$ & $N(H)$ & $\Gamma^{H}$ & $\operatorname{card} \Gamma^{H}$ & $\operatorname{dim} V^{H}$ & $\operatorname{dim} \Sigma_{[H]} / G$ & $\operatorname{dim} \Sigma_{[H]}$ \\
\hline Triclinic & $\mathbb{1}$ & $\mathrm{SO}(3)$ & $\mathrm{SO}(3)$ & $\infty$ & 9 & 6 & 9 \\
Monoclinic & $\mathbb{Z}_{2}$ & $\mathrm{O}(2)$ & $\mathrm{O}(2)$ & $\infty$ & 5 & 4 & 7 \\
Orthotropic & $\mathbb{D}_{2}$ & $\mathbb{O}$ & $\mathfrak{S}_{3}$ & 6 & 3 & 3 & 6 \\
Trigonal & $\mathbb{D}_{3}$ & $\mathbb{D}_{6}$ & $\mathfrak{S}_{2}$ & 2 & 2 & 2 & 5 \\
Tetragonal & $\mathbb{D}_{4}$ & $\mathbb{D}_{8}$ & $\mathfrak{S}_{2}$ & 2 & 2 & 2 & 5 \\
Transverse isotropic & $\mathrm{O}(2)$ & $\mathrm{O}(2)$ & $\mathbb{1}$ & 1 & 1 & 1 & 3 \\
Cubic & $\mathbb{O}$ & $\mathbb{O}$ & $\mathbb{1}$ & 1 & 1 & 1 & 4 \\
Isotropic & $\mathrm{SO}(3)$ & $\mathrm{SO}(3)$ & $\mathbb{1}$ & 1 & 0 & 0 & 0 \\
\hline
\end{tabular}

for $\mathbb{Z}_{3}, \mathbb{Z}_{4}$ (which are not isotropy subgroups). This may be why it was believed, for such a long time, that there was ten types of elasticity classes rather than eight.

Remark 3.7. The stratum $\Sigma_{[H]}$ consists of tensors whose symmetry group is conjugate to $H$. It corresponds to a symmetry class according to Forte and Vianello [17]. Formula (6) can be recast as

$$
\operatorname{dim} \Sigma_{[H]}=\operatorname{dim} V^{H}+e_{H}
$$

where $e_{H}=\operatorname{dim} G / N(H)$ is the Euler number ${ }^{8}$. In elasticity, $e_{H}$ is the number of Euler angles needed to define the appropriate coordinate system in which the elastic tensor has the minimum number of elastic constants [22]. The strata dimension corresponds to what Norris, for example, calls the number of independent parameters characterizing a tensor.

Remark 3.8. When the monodromy group $\Gamma^{H}$ is finite, $\Sigma_{[H]} / G$ and $V^{H}$ have the same dimension, this is the situation for most of elasticity symmetry classes. At the opposite, when $\Gamma^{H}$ is continuous, the dimension of the orbit space $\Sigma_{[H]} / G$ is strictly smaller than $\operatorname{dim} V^{H}$. In elasticity, this case occurs only for classes $\left[\mathbb{Z}_{2}\right]$ and $[\mathbb{1}]$.

The isotropy classes of the 9-D space $\mathbb{H}^{4}$, the higher order component of the harmonic decomposition of $\mathbb{E}$ la, are the same as the 8 classes of $\mathbb{E}$ la. The main characteristics of each symmetry classes are reported in the table 2.

\footnotetext{
8 Notice that $G / N(H)$ is in bijection with the set of distinct subgroups of $G$ which are conjugate to $H$, that is $[H]$
} 


\section{Semialgebraic structure on orbit spaces}

\subsection{Polynomial invariants}

The linear action of the group $G$ on the $\mathbb{R}$-vector space $V$ extends naturally to the vector space of polynomial functions defined on $V$ with values in $\mathbb{R}$. This extension is given by

$$
(g \cdot P)(v):=P\left(g^{-1} \cdot v\right)
$$

for every polynomial function $P$ on $V$ and every vector $v \in V$. The set of all invariant polynomials is a sub-algebra of the algebra $\mathbb{R}[V]$ of polynomial functions defined on $V$. This invariant algebra is denoted by $\mathbb{R}[V]^{G}$.

When $G$ is a compact Lie group, this algebra $\mathbb{R}[V]^{G}$ is of finite type $[21,26]$. This means that it is possible to find a finite set of invariant polynomials $J_{1}, \ldots, J_{N}$ which generates $\mathbb{R}[V]^{G}$ (as an algebra). A minimal generating set is called an integrity basis. Notice that, in general, the invariant algebra is not free, which means that it is not always possible to find a minimal generator set $J_{1}, \ldots, J_{N}$ which are algebraically independent $^{9}$. The polynomial relations among $J_{1}, \ldots, J_{N}$ are called syzygies. The set of such relations is also finitely generated.

\subsection{Inequalities defining orbit spaces}

The orbit space $V / G$ of a linear representation $V$ of a compact Lie group $G$ can be given the structure of a semialgebraic set [13], that is a subset of $\mathbb{R}^{N}$ defined by a boolean combination of polynomial equations and inequalities over $\mathbb{R}$. A general explicit construction has been proposed in [27] [27]. Since invariant polynomial functions on $V$ separate the orbits [2, Appendix C], it is possible to find a finite set of polynomial invariants $\left\{J_{1}, \ldots, J_{N}\right\}$ which defines a polynomial mapping $J: V \rightarrow \mathbb{R}^{N}$ such that

$$
J(\mathbf{C})=J\left(\mathbf{C}^{\prime}\right) \quad \text { iff } \mathbf{C} \text { and } \mathbf{C}^{\prime} \text { are in the same orbit. }
$$

This way, a $G$-orbit can be identified with a point in $J(V) \subset \mathbb{R}^{N}$ and the orbit space is described by the equations and inequalities which defined the subset $J(V)$ of $\mathbb{R}^{N}$.

\subsection{Equations for closed strata}

Similarly, each closed stratum can be described by a finite set of polynomial equations and inequalities. Let $[H]$ be a fixed isotropy class and $\Sigma_{[H]} \subset V$ be the corresponding stratum. Let $\left\{J_{1}, J_{2}, \ldots, J_{N}\right\}$ be a finite set of invariant polynomials which separate the orbits of $G$. To obtain the defining equations for $\Sigma_{[H]}$, we fix a subgroup $H$ in the conjugacy class $[H]$ and choose linear coordinates $\left(x^{i}\right)_{1 \leq i \leq q}$ on $V^{H}$. Then, we

\footnotetext{
9 Polynomials $P_{1}, \ldots, P_{N}$ are said to be algebraically independent over $\mathbb{R}$ if the only polynomial in $N$ variables which satisfies $Q\left(P_{1}, \ldots, P_{N}\right)=0$ is the zero polynomial.
} 
evaluate $\left\{J_{1}, J_{2}, \ldots, J_{N}\right\}$ on $V^{H}$ (as polynomials in the $x^{i}$ ) and we try to obtain implicit equations on the $J_{k}$ for the parametric system

$$
J_{k}=P_{k}\left(x^{1}, \ldots x^{q}\right), \quad k=1, \ldots, N, \quad P_{k} \in \mathbb{R}\left[X_{1}, \ldots, X_{q}\right],
$$

satisfied by the restriction of the $J_{k}$ to $V^{H}$.

This is however a difficult task in general (one might consider [16] for a full discussion about the implicitization problem). Moreover, it could happen that the algebraic variety $V_{I}$ defined by such an implicit system is bigger than the variety $V_{P}$, defined by the parametric system (8). Besides, we are confronted to the difficulty that we work over $\mathbb{R}$ which is not algebraically closed.

To overcome these difficulties, we observe that the restrictions of $\left\{J_{1}, J_{2}, \ldots, J_{N}\right\}$ to $V^{H}$ are $\Gamma^{H}$-invariant and therefore can be expressed as polynomial expressions of some generators $\sigma_{1}, \ldots, \sigma_{r}$ of the invariant algebra of the monodromy group $\Gamma^{H}$ on $V^{H}$. This observation reduces the complexity of the problem. We consider first the implicitization problem for the parametric system

$$
J_{k}=p_{k}\left(\sigma_{1}, \ldots, \sigma_{r}\right), \quad k=1, \ldots, N, \quad p_{k} \in \mathbb{R}\left[X_{1}, \ldots, X_{r}\right] .
$$

To solve this problem, we use a Groebner basis [16]. The main observation is that, in each case we considered, the system (9) leads to rational solutions. More precisely, we obtained a system of implicit equations (syzygies) which characterizes the closed stratum $\bar{\Sigma}_{[H]}$

$$
S_{j}\left(J_{1}, J_{2}, \ldots, J_{N}\right)=0, \quad j=1, \ldots l \quad S_{j} \in \mathbb{R}\left[X_{1}, \ldots, X_{N}\right],
$$

and a system

$$
\sigma_{i}=R_{i}\left(J_{1}, J_{2}, \ldots, J_{N}\right), \quad i=1, \ldots, r \quad R_{i} \in \mathbb{R}\left(X_{1}, \ldots, X_{N}\right),
$$

which express the $\sigma_{i}$ as rational ${ }^{10}$ functions of $\left\{J_{1}, J_{2}, \ldots, J_{N}\right\}$ on the open stratum $\Sigma_{[H]}$.

Remark 4.1. Beware, however, that these rational expressions may not be unique. However if some $\sigma_{i}$ can be written as $P_{1} / Q_{1}$ as well as $P_{2} / Q_{2}$ then $P_{1} Q_{2}-P_{2} Q_{1}$ belongs to the ideal generated by the $S_{j}$.

Because the expressions of $\sigma_{i}$ are rational, the fact that the field on which we work is real or complex does not matter at this level. Therefore, each real solution $\left(J_{1}, J_{2}, \ldots, J_{N}\right)$ of $(10)$ corresponds to a unique real solution $\left(\sigma_{1}, \ldots, \sigma_{r}\right)$ given by (11).

The second step is to obtain a real point $\left(x^{1}, \ldots, x^{q}\right)$ in $V^{H}$ from a real solution $\left(\sigma_{1}, \ldots, \sigma_{r}\right)$ of $(11)$. For this, we need to compute an additional system of inequalities on the $\sigma_{i}$, or equivalently on the $J_{k}$, which permits to exclude complex solutions of

$$
\sigma_{i}=Q_{i}\left(x^{1}, \ldots x^{q}\right), \quad i=1, \ldots, r, \quad Q_{i} \in \mathbb{R}\left[X_{1}, \ldots, X_{q}\right] .
$$

In section 5 where our results are presented, the only non-trivial monodromy group that we encountered were the standard action of the symmetric groups $\mathfrak{S}_{2}$ and

\footnotetext{
10 The fact that the solutions are rational will not be justified here. We just observe that this is the case for all classes we have treated in this article.
} 
$\mathfrak{S}_{3}$ (in appropriate coordinates). In these cases, and more generally when the monodromy group is $\mathfrak{S}_{n}$ and the action is isomorphic to the standard one, $r=q=n$, the invariants $\sigma_{1}, \ldots, \sigma_{n}$ are algebraically independent and (in an appropriate coordinates system) $x^{1}, \ldots, x^{n}$ are the roots of the polynomial

$$
p(z)=z^{n}-\sigma_{1} z^{n-1}+\cdots+(-1)^{n} \sigma_{n} .
$$

Therefore, the problem reduces to finding conditions on the $\sigma_{i}$ that ensure that all the roots of $p$ are real. The solution is due to Hermite, [13]. He has proved that the number of distinct real roots of a real polynomial $p$ of degree $n$ is equal to the signature of the Hankel matrix $B(p):=\left(S_{i+j-2}\right)_{1 \leq i, j \leq n}$, where $S_{k}:=\sum_{i=1}^{n}\left(x^{i}\right)^{k}$ is the power sum of the roots of $p$. In particular, $p$ has real roots if and only if $B(p)$ is non-negative.

\section{Symmetry classes of $\mathbb{H}^{4}$}

Contrary to $\mathbb{E}$ la, an integrity basis for $\mathbb{H}^{4}$ is known ${ }^{11}$. The aim of this section is to describe the strata of $\mathbb{H}^{4}$, applying the algebraic procedure detailed in section 4.3 and using this integrity basis. We obtain a characterization, in terms of polynomial relations between invariants, for isotropy classes having finite monodromy group $\Gamma^{H}$, namely the cubic class $[\mathbb{O}]$, the transversely isotropic class $[\mathrm{O}(2)]$, the trigonal class $\left[\mathbb{D}_{3}\right]$, the tetragonal class $\left[\mathbb{D}_{4}\right]$ and the orthotropic class $\left[\mathbb{D}_{2}\right]$. For each of them, explicit polynomial relations between the elementary invariant of $\mathbb{H}^{4}$ is given. Finally, a bifurcation diagram is provided to make explicit how we "travel" from a given isotropy class to another.

Let $D \in \mathbb{H}^{4}$, we introduce the following 2 nd-order tensors $\mathbf{d}_{2}, \ldots, \mathbf{d}_{10}$ (which are covariant $^{12}$ to $D$ )

$$
\begin{array}{llc}
\mathbf{d}_{2}=\operatorname{tr}_{13}\left(D^{2}\right) & \mathbf{d}_{3}=\operatorname{tr}_{13}\left(D^{3}\right) & \mathbf{d}_{4}=\mathbf{d}_{2}^{2} \\
\mathbf{d}_{5}=\mathbf{d}_{2} D \mathbf{d}_{2} & \mathbf{d}_{6}=\mathbf{d}_{2}^{3} & \mathbf{d}_{7}=\mathbf{d}_{2}^{2} D \mathbf{d}_{2} \\
\mathbf{d}_{8}=\mathbf{d}_{2}^{2} D^{2} \mathbf{d}_{2} & \mathbf{d}_{9}=\mathbf{d}_{2}^{2} D \mathbf{d}_{2}^{2} & \mathbf{d}_{10}=\mathbf{d}_{2}^{2} D^{2} \mathbf{d}_{2}^{2}
\end{array}
$$

Proposition 5.1. An integrity basis for $\mathbb{H}^{4}$ is given by

$$
J_{k}:=\operatorname{tr}\left(\mathbf{d}_{k}\right), \quad k=2, \ldots, 10 .
$$

The first six invariants $J_{2}, \ldots, J_{7}$ are algebraically independent. The last three ones $J_{8}, J_{9}, J_{10}$ are linked to the formers by polynomial relations. These fundamental syzygies were computed in [32]. For technical details and historical considerations concerning these fundamental invariants, we refer to the original publication of Boelher and al.[8] and the references therein.

Remark 5.2. Notice that the first invariant $J_{2}(D)=\langle D, D\rangle$ is the squared norm of $D$, it corresponds to the squared Frobenius norm of $D$. In particular, $J_{2}(D)=0$ if and only if $D=0$.

\footnotetext{
11 This integrity basis has been computed in [32] and brings to the knowledge of the mechanic community in [8].

12 A linear map $\Phi: V_{1} \rightarrow V_{2}$, between two representations $\left(V_{1}, \rho_{1}\right)$ and $\left(V_{2}, \rho_{2}\right)$ of a same group $G$ is equivariant if $\Phi\left(\rho_{1}(g) \cdot v_{1}\right)=\rho_{2}(g) \cdot \Phi\left(v_{1}\right)$, for all $v_{1} \in V_{1}$ and $g \in G$. In that case, we say that $v_{2}:=\Phi\left(v_{1}\right)$ is covariant to $v_{1}$.
} 
5.1 Cubic symmetry $([\mathbb{O}])$

An $\mathbb{O}$-invariant tensor $D \in \mathbb{H}^{4}$ has the following matrix representation:

$$
\underline{D}=\left(\begin{array}{cccccc}
8 \delta & -4 \delta & -4 \delta & 0 & 0 & 0 \\
-4 \delta & 8 \delta & -4 \delta & 0 & 0 & 0 \\
-4 \delta & -4 \delta & 8 \delta & 0 & 0 & 0 \\
0 & 0 & 0 & -8 \delta & 0 & 0 \\
0 & 0 & 0 & 0 & -8 \delta & 0 \\
0 & 0 & 0 & 0 & 0 & -8 \delta
\end{array}\right)
$$

where $\delta \in \mathbb{R}$.

As indicated in Table 1, the monodromy group for the cubic system is $\mathbb{1}$, therefore linear slice corresponding to the octahedral group is of degree 1 . In other terms, there is no ambiguity in defining the normal form (13). The evaluation of the invariants (12) on this slice gives the following parametric system

$$
\begin{aligned}
& J_{2}=480 \delta^{2}, \quad J_{3}=1920 \delta^{3}, \quad J_{4}=76800 \delta^{4}, \\
& J_{5}=0, \quad J_{6}=12288000 \delta^{6}, \quad J_{7}=0, \\
& J_{8}=0, \quad J_{9}=0, \quad J_{10}=0 .
\end{aligned}
$$

We notice that the parameter $4 \delta=J_{3} / J_{2}$ is a rational invariant.

Proposition 5.3. A harmonic tensor $D \in \mathbb{H}^{4}$ is in the closed stratum $\bar{\Sigma}_{[\mathbb{O}]}$ if and only if the invariants $J_{2}(D) \cdots J_{10}(D)$ satisfy the following polynomial relations

$$
\begin{aligned}
& 3 J_{4}=J_{2}{ }^{2}, \quad J_{5}=0, \quad 30 J_{3}{ }^{2}=J_{2}{ }^{3}, \quad 9 J_{6}=J_{2}{ }^{3}, \\
& J_{7}=0, \quad J_{8}=0, \quad J_{9}=0, \quad J_{10}=0 .
\end{aligned}
$$

$D$ is in the cubic class $[\mathbb{O}]$ if moreover $J_{2}(D) \neq 0$. In that case, it admits the normal form (13) where $4 \delta:=J_{3}(D) / J_{2}(D)$.

We observe that $\forall J_{2}, J_{3} \in \mathbb{R}, \delta \in \mathbb{R}$. Therefore we do not need to add any inequality to the syzygy system (14) to ensure that $\delta$ is real. This fact is general to any linear slice of degree 1 .

Remark 5.4. All 2nd-order tensors in $\mathbb{H}^{2}$ covariant to $D$ vanish.

As a consequence, for the cubic case, the following characterization is possible.

Corollary 5.5. A harmonic tensor $D \in \mathbb{H}^{4}$ is in the closed stratum $\bar{\Sigma}_{[\mathbb{O}]}$ if and only if there exists $c_{2}, c_{3} \in \mathbb{R}^{\star}$ such that

$$
\mathbf{d}_{2}=c_{2} \mathbf{q}, \quad \mathbf{d}_{3}=c_{3} \mathbf{q}, \quad \text { and } \quad 10 c_{3}^{2}-c_{2}^{3}=0 .
$$

Proof. Since $\mathbf{d}_{k}$ are covariant to an $\mathbb{O}$-invariant tensor, they are multiple of the identity, i.e., $\mathbf{d}_{k}=c_{k} \mathbf{q}$, for $k=2, \ldots, 10$. Thus $J_{k}=\operatorname{tr}\left(\mathbf{d}_{k}\right)=3 c_{k}$ and the set of syzygies (14) can be expressed in terms of the $c_{k}$. The announced relations follow, using the additional fact that $D \mathbf{q}=0$ and the definitions of the $\mathbf{d}_{k}$.

Conversely, suppose that these relations are verified. The fundamental $D$ covariants, provided in proposition (5.1), are either $\mathbf{d}_{3}$, powers of $\mathbf{d}_{2}$, or involved products of 
$D$ and $\mathbf{d}_{2}$. If $\mathbf{d}_{2}=c_{2} \mathbf{q}$, powers of $\mathbf{d}_{2}$ are scalar multiples of the metric $\mathbf{q}$, and because $D$ is harmonic all terms involving $D \mathbf{d}_{2}$ vanish. Therefore $J_{5}, J_{7}, J_{8}, J_{9}, J_{10}$ vanish and the relations between $J_{2}, J_{4}$ and $J_{6}$ are automatically satisfied. Because $\operatorname{tr} \mathbf{q}=3$ and $10 c_{3}^{2}-c_{2}^{3}=0$, we have moreover $10 J_{3}^{2}-J_{2}^{3}=0$. Therefore the fundamental syzygies of the cubic class are satisfied.

5.2 Transversely isotropic symmetry $([\mathrm{O}(2)])$

An $\mathrm{O}(2)$-invariant tensor $D \in \mathbb{H}^{4}$ has the following matrix representation:

$$
\underline{D}=\left(\begin{array}{cccccc}
3 \delta & \delta & -4 \delta & 0 & 0 & 0 \\
\delta & 3 \delta & -4 \delta & 0 & 0 & 0 \\
-4 \delta & -4 \delta & 8 \delta & 0 & 0 & 0 \\
0 & 0 & 0 & -8 \delta & 0 & 0 \\
0 & 0 & 0 & 0 & -8 \delta & 0 \\
0 & 0 & 0 & 0 & 0 & 2 \delta
\end{array}\right)
$$

where $\delta \in \mathbb{R}$.

As in the previous case, the monodromy group for the transversely isotropic symmetry is $\mathbb{1}$, and the linear slice is of degree 1 . The evaluation of the invariants (12) on this slice gives

$$
\begin{aligned}
& J_{2}=280 \delta^{2}, \quad J_{3}=720 \delta^{3}, \quad J_{4}=32800 \delta^{4}, \\
& J_{5}=80000 \delta^{5}, \quad J_{6}=4528000 \delta^{6}, \quad J_{7}=17600000 \delta^{7}, \\
& J_{8}=211200000 \delta^{8}, \quad J_{9}=3872000000 \delta^{9}, \quad J_{10}=46464000000 \delta^{10}
\end{aligned}
$$

The parameter $\delta=7 J_{3} / 18 J_{2}$ is a rational invariant. The linear slice corresponding to the group $\mathrm{O}(2)$ is of degree 1 , which was already known (see section $\mathrm{A}$ ).

Proposition 5.6. A harmonic tensor $D \in \mathbb{H}^{4}$ is in the closed stratum $\bar{\Sigma}_{[\mathrm{O}(2)]}$ if and only if the invariants $J_{2}(D) \cdots J_{10}(D)$ satisfy the following polynomial relations

$$
\begin{aligned}
& 98 J_{4}=41 J_{2}{ }^{2}, \quad 63 J_{5}=25 J_{3} J_{2}, \quad 3430 J_{3}{ }^{2}=81 J_{2}{ }^{3}, \\
& 1372 J_{6}=283 J_{2}{ }^{3}, \quad 882 J_{7}=275 J_{2}{ }^{2} J_{3}, \quad 4802 J_{8}=165 J_{2}{ }^{4}, \\
& 12348 J_{9}=3025 J_{2}{ }^{3} J_{3}, \quad 67228 J_{10}=1815 J_{2}{ }^{5} .
\end{aligned}
$$

$D$ is in the transversely isotropic class $[\mathrm{O}(2)]$ if moreover $J_{2}(D) \neq 0$. In that case, it admits the normal form (16) where $\delta=7 J_{3}(D) / 18 J_{2}(D)$. real.

As for $[\mathbb{O}]$ the expression of $\delta$ ensures that, for all $J_{2}, J_{3} \in \mathbb{R}$, the solution $\delta$ is

Remark 5.7. All 2nd-order tensors in $\mathbb{H}^{2}$ covariant to $D$ are multiple of diag $(1,1,-2)$ and are eigenvectors of $D$ corresponding to the eigenvalue $12 \delta$. 
5.3 Trigonal symmetry $\left(\left[\mathbb{D}_{3}\right]\right)$

A $\mathbb{D}_{3}$-invariant tensor $D \in \mathbb{H}^{4}$ has the following matrix representation:

$$
\underline{D}=\left(\begin{array}{cccccc}
3 \delta & \delta & -4 \delta & -\sqrt{2} \sigma & 0 & 0 \\
\delta & 3 \delta & -4 \delta & \sqrt{2} \sigma & 0 & 0 \\
-4 \delta & -4 \delta & 8 \delta & 0 & 0 & 0 \\
-\sqrt{2} \sigma & \sqrt{2} \sigma & 0 & -8 \delta & 0 & 0 \\
0 & 0 & 0 & 0 & -8 \delta & -2 \sigma \\
0 & 0 & 0 & 0 & -2 \sigma & 2 \delta
\end{array}\right)
$$

where $(\delta, \sigma) \in \mathbb{R}^{2}$.

As indicated in the table 1 for this symmetry class is no more $\mathbb{1}$ but $\mathfrak{S}_{2}$, and the linear slice is of degree 2 . Its action is given by

$$
\delta \mapsto \delta, \quad \sigma \mapsto-\sigma .
$$

Therefore, we will have to make a choice between $\sigma$ and $-\sigma$ to define our normal form. The evaluation of the invariants (12) on this slice gives

$$
\begin{aligned}
& J_{2}=280 \delta^{2}+16 \sigma^{2} \\
& J_{3}=144 \delta\left(5 \delta^{2}-\sigma^{2}\right) \\
& J_{4}=32800 \delta^{4}+2720 \sigma^{2} \delta^{2}+88 \sigma^{4} \\
& J_{5}=32 \delta\left(-\sigma^{2}+50 \delta^{2}\right)^{2} \\
& J_{6}=4528000 \delta^{6}+436800 \sigma^{2} \delta^{4}+20640 \sigma^{4} \delta^{2}+496 \sigma^{6} \\
& J_{7}=320 \delta\left(22 \delta^{2}+\sigma^{2}\right)\left(-\sigma^{2}+50 \delta^{2}\right)^{2} \\
& J_{8}=3840 \delta^{2}\left(22 \delta^{2}+\sigma^{2}\right)\left(-\sigma^{2}+50 \delta^{2}\right)^{2} \\
& J_{9}=3200 \delta\left(-\sigma^{2}+50 \delta^{2}\right)^{2}\left(22 \delta^{2}+\sigma^{2}\right)^{2} \\
& J_{10}=38400 \delta^{2}\left(-\sigma^{2}+50 \delta^{2}\right)^{2}\left(22 \delta^{2}+\sigma^{2}\right)^{2}
\end{aligned}
$$

We notice that the parameter:

$$
\delta=-\frac{1}{4} \frac{J_{5}}{J_{2}^{2}-3 J_{4}}
$$

is a rational invariant, therefore for all $J_{2}, J_{4}, J_{5} \in \mathbb{R}$, the parameter $\delta$ is real (c.f. section 4.3). However, the minimal equation satisfied by $\sigma$ is of degree 2

$$
J_{2}=280 \delta^{2}+16 \sigma^{2} \text {. }
$$

A condition is needed for $\sigma$ to be real. This condition, which is $\sigma^{2} \geq 0$, is given by the following inequality on $J_{2}, J_{4}, J_{5}$ :

$$
2 J_{2}\left(J_{2}^{2}-3 J_{4}\right)^{2}-35 J_{5}^{2} \geq 0
$$

Having computed $\delta$, the equation (19) has two roots with opposite signs. 
Proposition 5.8. A harmonic tensor $D \in \mathbb{H}^{4}$ is in the closed stratum $\bar{\Sigma}_{\left[\mathbb{D}_{3}\right]}$ if and only if the invariants $J_{2}(D) \cdots J_{10}(D)$ satisfy the following polynomial relations

$$
\begin{aligned}
192 J_{6} & =-51 J_{2}{ }^{3}+216 J_{2} J_{4}+10 J_{3}{ }^{2} \\
36 J_{7} & =-2 J_{2}{ }^{2} J_{3}+6 J_{3} J_{4}+27 J_{2} J_{5} \\
768 J_{4}{ }^{2} & =-99 J_{2}{ }^{4}+552 J_{2}{ }^{2} J_{4}+10 J_{2} J_{3}^{2}+240 J_{3} J_{5} \\
240 J_{8} & =-33 J_{2}{ }^{4}+96 J_{2}{ }^{2} J_{4}+30 J_{2} J_{3}^{2}+40 J_{3} J_{5} \\
576 J_{4} J_{5} & =-41 J_{2}{ }^{3} J_{3}+120 J_{2} J_{3} J_{4}+216 J_{2}{ }^{2} J_{5}+30 J_{3}{ }^{3} \\
1152 J_{9} & =-99 J_{2}{ }^{3} J_{3}+296 J_{2} J_{3} J_{4}+648 J_{2}^{2} J_{5}+10 J_{3}{ }^{3} \\
1440 J_{5} & =-11 J_{2}{ }^{5}+32 J_{2}{ }^{3} J_{4}-70 J_{2}{ }^{2} J_{3}{ }^{2}+240 J_{2} J_{3} J_{5}+240 J_{3}{ }^{2} J_{4} \\
8640 J_{10} & =-891 J_{2}{ }^{5}+2592 J_{2}{ }^{3} J_{4}+730 J_{2}{ }^{2} J_{3}{ }^{2}+2160 J_{2} J_{3} J_{5}+240 J_{3}{ }^{2} J_{4}
\end{aligned}
$$

together with inequality (20). $D$ is in the trigonal class $\left[\mathbb{D}_{3}\right]$ if moreover $3 J_{4}-J_{2}^{2} \neq 0$ and $98 J_{4}-41 J_{2}^{2} \neq 0$. In that case, it admits the normal form (18) where

$$
\delta=-\frac{1}{4} \frac{J_{5}}{J_{2}^{2}-3 J_{4}}
$$

and where $\sigma$ is the positive root of $J_{2}=280 \delta^{2}+16 \sigma^{2}$.

Remark 5.9. All 2 nd-order tensors in $\mathbb{H}^{2}$ covariant to $D$ are multiple of diag $(1,1,-2)$ and are eigenvectors of $D$ corresponding to the eigenvalue $12 \delta$.

\subsection{Tetragonal symmetry $\left(\left[\mathbb{D}_{4}\right]\right)$}

A $\mathbb{D}_{4}$-invariant tensor $D \in \mathbb{H}^{4}$ has the following matrix representation:

$$
\underline{D}=\left(\begin{array}{cccccc}
-\sigma+3 \delta & \sigma+\delta & -4 \delta & 0 & 0 & 0 \\
\sigma+\delta & -\sigma+3 \delta & -4 \delta & 0 & 0 & 0 \\
-4 \delta & -4 \delta & 8 \delta & 0 & 0 & 0 \\
0 & 0 & 0 & -8 \delta & 0 & 0 \\
0 & 0 & 0 & 0 & -8 \delta & 0 \\
0 & 0 & 0 & 0 & 0 & 2 \sigma+2 \delta
\end{array}\right)
$$

where $(\delta, \sigma) \in \mathbb{R}^{2}$. As for the $\left[\mathbb{D}_{3}\right]$ class, the monodromy group is $\mathfrak{S}_{2}$, and the linear slice is of degree 2 . Therefore a choice has to be made between $\sigma$ and $-\sigma$ to define the normal form. 
The evaluation of the invariants (12) on this slice gives

$$
\begin{aligned}
& J_{2}=8 \sigma^{2}+280 \delta^{2} \\
& J_{3}=48 \delta\left(\sigma^{2}+15 \delta^{2}\right) \\
& J_{4}=32 \sigma^{4}+960 \delta^{2} \sigma^{2}+32800 \delta^{4} \\
& J_{5}=128 \delta(5 \delta-\sigma)^{2}(5 \delta+\sigma)^{2} \\
& J_{6}=128 \sigma^{6}+5760 \sigma^{4} \delta^{2}+86400 \sigma^{2} \delta^{4}+4528000 \delta^{6} \\
& J_{7}=512 \delta\left(55 \delta^{2}+\sigma^{2}\right)(5 \delta-\sigma)^{2}(5 \delta+\sigma)^{2} \\
& J_{8}=6144 \delta^{2}\left(55 \delta^{2}+\sigma^{2}\right)(5 \delta-\sigma)^{2}(5 \delta+\sigma)^{2} \\
& J_{9}=2048 \delta(5 \delta-\sigma)^{2}(5 \delta+\sigma)^{2}\left(55 \delta^{2}+\sigma^{2}\right)^{2} \\
& J_{10}=24576 \delta^{2}(5 \delta-\sigma)^{2}(5 \delta+\sigma)^{2}\left(55 \delta^{2}+\sigma^{2}\right)^{2}
\end{aligned}
$$

We notice that the parameter

$$
\delta=-\frac{1}{4} \frac{J_{5}}{J_{2}^{2}-3 J_{4}}
$$

is a rational invariant, therefore for all $\left(J_{2}, J_{4}, J_{5}\right) \in \mathbb{R}^{3}, \delta \in \mathbb{R}$. However, the minimal equation satisfied by $\sigma$ is of degree 2

$$
J_{2}=8 \sigma^{2}+280 \delta^{2}
$$

As for $\left[\mathbb{D}_{3}\right]$, the condition

$$
2 J_{2}\left(J_{2}^{2}-3 J_{4}\right)^{2}-35 J_{5}^{2} \geq 0
$$

has to be added in order to ensure $\sigma$ to be real.

Having computed $\delta$, the equation (23) has two roots with opposite signs. The linear slice corresponding to the group $\mathbb{D}_{4}$ is of degree 2 , which was already known (see section A).

Proposition 5.10. A harmonic tensor $D \in \mathbb{H}^{4}$ is in the closed stratum $\bar{\Sigma}_{\left[\mathbb{D}_{4}\right]}$ if and only if the invariants $J_{2}(D) \cdots J_{10}(D)$ satisfy the following polynomial relations

$$
\begin{aligned}
6 J_{6} & =-3 J_{2}{ }^{3}+9 J_{2} J_{4}+20 J_{3}{ }^{2} \\
3 J_{7} & =J_{2}{ }^{2} J_{3}-3 J_{3} J_{4}+3 J_{2} J_{5} \\
6 J_{4}{ }^{2} & =-3 J_{2}{ }^{4}+9 J_{2}{ }^{2} J_{4}+20 J_{2} J_{3}^{2}-20 J_{3} J_{5} \\
5 J_{8} & =-3 J_{2}{ }^{4}+6 J_{2}{ }^{2} J_{4}+30 J_{2} J_{3}{ }^{2}-5 J_{3} J_{5} \\
3 J_{4} J_{5} & =7 J_{2}{ }^{3} J_{3}-15 J_{2} J_{3} J_{4}+3 J_{2}{ }^{2} J_{5}-60 J_{3}{ }^{3} \\
6 J_{9} & =5 J_{2}{ }^{3} J_{3}-13 J_{2} J_{3} J_{4}+6 J_{2}{ }^{2} J_{5}-20 J_{3}{ }^{3} \\
5 J_{5}{ }^{2} & =-2 J_{2}{ }^{5}+4 J_{2}{ }^{3} J_{4}+10 J_{2}{ }^{2} J_{3}{ }^{2}-20 J_{2} J_{3} J_{5}+30 J_{3}{ }^{2} J_{4} \\
15 J_{10} & =-9 J_{2}{ }^{5}+18 J_{2}{ }^{3} J_{4}+85 J_{2}{ }^{2} J_{3}{ }^{2}-30 J_{2} J_{3} J_{5}+15 J_{3}{ }^{2} J_{4}
\end{aligned}
$$


together with inequality (24). $D$ is in the tetragonal class $\left[\mathbb{D}_{4}\right]$ if moreover $3 J_{4}-J_{2}^{2} \neq$ 0 and $5 J_{2}^{3}-8 J_{2} J_{4}-70 J_{3}^{2} \neq 0$. In that case, it admits the normal form (22) where

$$
\delta=-\frac{1}{4} \frac{J_{5}}{J_{2}^{2}-3 J_{4}}
$$

and where $\sigma$ is the positive root of $J_{2}=8 \sigma^{2}+280 \delta^{2}$.

Remark 5.11. All 2nd-order tensors in $\mathbb{H}^{2}$ covariant to $D$ are multiple of diag $(1,1,-2)$ and are eigenvectors of $D$ corresponding to the eigenvalue $12 \delta$.

5.5 Orthotropic symmetry $\left(\left[\mathbb{D}_{2}\right]\right)$

A $\mathbb{D}_{2}$-invariant tensor $D \in \mathbb{H}^{4}$ has the following matrix representation:

$$
\underline{D}=\left(\begin{array}{cccccc}
-\lambda_{2}-\lambda_{3} & \lambda_{3} & \lambda_{2} & 0 & 0 & 0 \\
\lambda_{3} & -\lambda_{3}-\lambda_{1} & \lambda_{1} & 0 & 0 & 0 \\
\lambda_{2} & \lambda_{1} & -\lambda_{1}-\lambda_{2} & 0 & 0 & 0 \\
0 & 0 & 0 & 2 \lambda_{1} & 0 & 0 \\
0 & 0 & 0 & 0 & 2 \lambda_{2} & 0 \\
0 & 0 & 0 & 0 & 0 & 2 \lambda_{3}
\end{array}\right)
$$

where $\left(\lambda_{1}, \lambda_{2}, \lambda_{3}\right) \in \mathbb{R}^{3}$. The monodromy group is the group $\mathfrak{S}_{3}$ acting on $\left(\lambda_{1}, \lambda_{2}, \lambda_{3}\right)$ by permutation, therefore the linear slice is of degree 6 . This fact will make the following computation rather cumbersome. As the evaluations of the invariants (12) on this slice are $\mathfrak{S}_{3}$-invariant, they can therefore be expressed as polynomial functions of the elementary symmetric polynomials $\left(\sigma_{1}, \sigma_{2}, \sigma_{3}\right)$ defined by:

$$
\sigma_{1}:=\lambda_{1}+\lambda_{2}+\lambda_{3}, \quad \sigma_{2}:=\lambda_{1} \lambda_{2}+\lambda_{1} \lambda_{3}+\lambda_{2} \lambda_{3}, \quad \sigma_{3}:=\lambda_{1} \lambda_{2} \lambda_{3} .
$$


More precisely, we have:

$$
\begin{aligned}
J_{2}= & -14 \sigma_{2}+8 \sigma_{1}^{2} \\
J_{3}= & -6 \sigma_{1} \sigma_{2}+24 \sigma_{3} \\
J_{4}= & 40 \sigma_{1} \sigma_{3}-112 \sigma_{1}^{2} \sigma_{2}+68 \sigma_{2}^{2}+32 \sigma_{1}^{4} \\
J_{5}= & 64 \sigma_{1}^{2} \sigma_{3}-12 \sigma_{2} \sigma_{3}-16 \sigma_{1}^{3} \sigma_{2}+28 \sigma_{1} \sigma_{2}^{2} \\
J_{6}= & -344 \sigma_{2}^{3}+192 \sigma_{1}^{3} \sigma_{3}-24 \sigma_{3}^{2}-672 \sigma_{1}^{4} \sigma_{2}+1008 \sigma_{1}^{2} \sigma_{2}^{2} \\
& +128 \sigma_{1}^{6}-504 \sigma_{1} \sigma_{2} \sigma_{3} \\
J_{7}= & -432 \sigma_{1}^{2} \sigma_{2} \sigma_{3}+384 \sigma_{1}^{4} \sigma_{3}+104 \sigma_{2}^{2} \sigma_{3}-96 \sigma_{1} \sigma_{3}^{2}-64 \sigma_{1}^{5} \sigma_{2} \\
& +192 \sigma_{1}^{3} \sigma_{2}^{2}-248 \sigma_{1} \sigma_{2}^{3} \\
J_{8}= & 608 \sigma_{1}^{3} \sigma_{2} \sigma_{3}+80 \sigma_{2}^{4}-768 \sigma_{1}^{5} \sigma_{3}+192 \sigma_{1}^{2} \sigma_{3}^{2}+72 \sigma_{2} \sigma_{3}^{2} \\
& +288 \sigma_{1}^{4} \sigma_{2}^{2}-416 \sigma_{1}^{2} \sigma_{2}^{3}+744 \sigma_{1} \sigma_{2}^{2} \sigma_{3} \\
J_{9}= & -5248 \sigma_{1}^{4} \sigma_{2} \sigma_{3}+2880 \sigma_{1}^{2} \sigma_{2}^{2} \sigma_{3}+1328 \sigma_{1} \sigma_{2} \sigma_{3}^{2}+144 \sigma_{3}^{3}+2304 \sigma_{1}^{6} \sigma_{3} \\
& -1152 \sigma_{1}^{3} \sigma_{3}^{2}-880 \sigma_{2}^{3} \sigma_{3}-256 \sigma_{1}^{7} \sigma_{2}+1024 \sigma_{1}^{5} \sigma_{2}^{2}-2304 \sigma_{1}^{3} \sigma_{2}^{3}+2160 \sigma_{1} \sigma_{2}^{4} \\
J_{10}= & 10752 \sigma_{1}^{5} \sigma_{2} \sigma_{3}-1280 \sigma_{1}^{3} \sigma_{2}^{2} \sigma_{3}-5664 \sigma_{1} \sigma_{2}^{3} \sigma_{3}-2688 \sigma_{1}^{2} \sigma_{2} \sigma_{3}^{2}-800 \sigma_{2}^{5} \\
& -4608 \sigma_{1}^{7} \sigma_{3}+2304 \sigma_{1}^{4} \sigma_{3}^{2}-1344 \sigma_{2}^{2} \sigma_{3}^{2}-288 \sigma_{1} \sigma_{3}^{3}+1536 \sigma_{1}^{6} \sigma_{2}^{2} \\
& -4224 \sigma_{1}^{4} \sigma_{2}^{3}+3104 \sigma_{1}^{2} \sigma_{2}^{4}
\end{aligned}
$$

As it can be observed, the parametric system obtained for $\left[\mathbb{D}_{2}\right]$ is rather complicated. Nevertheless, the evaluation of $J_{2}, \ldots, J_{10}$ on the slice and their expressions using $\sigma_{1}$, $\sigma_{2}, \sigma_{3}$ is simple using a computer. By computing the elimination ideal of a Groebner basis for the ideal generated by

$$
J_{k}-p_{k}\left(\sigma_{1}, \sigma_{2}, \sigma_{3}\right), \quad k=2, \ldots, 7
$$

we obtain a set of 6 syzygies.

$$
\begin{aligned}
&-1350 J_{3} J_{7}-840 J_{4} J_{6}+ 465 J_{2}{ }^{2} J_{6}+270 J_{5}{ }^{2}+720 J_{2} J_{3} J_{5}+747 J_{2} J_{4}{ }^{2} \\
&-170 J_{3}{ }^{2} J_{4}-564 J_{2}{ }^{3} J_{4}+70 J_{2}{ }^{2} J_{3}{ }^{2}+84 J_{2}{ }^{5}=0 \\
&-1620 J_{4} J_{7}+810 J_{2}{ }^{2} J_{7}+360 J_{5} J_{6}-1110 J_{2} J_{3} J_{6}+999 J_{2} J_{4} J_{5}+960 J_{3}{ }^{2} J_{5} \\
&-549 J_{2}{ }^{3} J_{5}-972 J_{3} J_{4}{ }^{2}+1638 J_{2}{ }^{2} J_{3} J_{4}-80 J_{2} J_{3}{ }^{3}-312 J_{2}{ }^{4} J_{3}=0
\end{aligned}
$$

$$
\begin{gathered}
4050 J_{5} J_{7}-25650 J_{2} J_{3} J_{7}-14310 J_{2} J_{4} J_{6}+9600 J_{3}{ }^{2} J_{6}+7965 J_{2}{ }^{3} J_{6}+9450 J_{3} J_{4} J_{5} \\
+10530 J_{2}{ }^{2} J_{3} J_{5}+1134 J_{4}{ }^{3}+11259 J_{2}{ }^{2} J_{4}{ }^{2}-12330 J_{2} J_{3}{ }^{2} J_{4}-9018 J_{2}{ }^{4} J_{4} \\
+400 J_{3}{ }^{4}+3270 J_{2}{ }^{3} J_{3}{ }^{2}+1350 J_{2}{ }^{6}=0 \quad(27 \mathrm{c})
\end{gathered}
$$




$$
\begin{gathered}
-12150 J_{2} J_{3} J_{7}+3600 J_{6}{ }^{2}-11610 J_{2} J_{4} J_{6}+9750 J_{3}{ }^{2} J_{6}+4410 J_{2}{ }^{3} J_{6} \\
+8505 J_{3} J_{4} J_{5}+3645 J_{2}{ }^{2} J_{3} J_{5}+1458 J_{4}{ }^{3}+5670 J_{2}{ }^{2} J_{4}{ }^{2} \\
-10710 J_{2} J_{3}{ }^{2} J_{4}-4104 J_{2}{ }^{4} J_{4}+400 J_{3}{ }^{4}+2580 J_{2}{ }^{3} J_{3}{ }^{2}+576 J_{2}{ }^{6}=0
\end{gathered}
$$

$$
\begin{gathered}
1800 J_{6} J_{7}-10800 J_{2} J_{4} J_{7}+4800 J_{3}{ }^{2} J_{7}+4950 J_{2}{ }^{3} J_{7}+4020 J_{3} J_{4} J_{6}-8370 J_{2}{ }^{2} J_{3} J_{6} \\
+162 J_{4}{ }^{2} J_{5}+7371 J_{2}{ }^{2} J_{4} J_{5}+2880 J_{2} J_{3}{ }^{2} J_{5}-3483 J_{2}{ }^{4} J_{5}-9216 J_{2} J_{3} J_{4}{ }^{2} \\
+640 J_{3}{ }^{3} J_{4}+11946 J_{2}{ }^{3} J_{3} J_{4}-720 J_{2}{ }^{2} J_{3}{ }^{3}-2160 J_{2}{ }^{5} J_{3}=0
\end{gathered}
$$

$$
\begin{array}{r}
60750 J_{7}{ }^{2}+178200 J_{3} J_{4} J_{7}-546750 J_{2}{ }^{2} J_{3} J_{7}+3780 J_{4}{ }^{2} J_{6}-246780 J_{2}{ }^{2} J_{4} J_{6} \\
+348000 J_{2} J_{3}{ }^{2} J_{6}+137025 J_{2}{ }^{4} J_{6}+116640 J_{2} J_{3} J_{4} J_{5}-75600 J_{3}{ }^{3} J_{5} \\
+223560 J_{2}{ }^{3} J_{3} J_{5}+29808 J_{2} J_{4}{ }^{3}+82170 J_{3}{ }^{2} J_{4}{ }^{2} \\
+177660 J_{2}{ }^{3} J_{4}{ }^{2}-438390 J_{2}{ }^{2} J_{3}{ }^{2} J_{4}-148014 J_{2}{ }^{5} J_{4} \\
+17200 J_{2} J_{3}{ }^{4}+102000 J_{2}{ }^{4} J_{3}{ }^{2}+22221 J_{2}{ }^{7}=0
\end{array}
$$

If these relations are satisfied, the elementary symmetric polynomials $\sigma_{1}, \sigma_{2}$ and $\sigma_{3}$ can be expressed, on the open stratum defined by

$$
6 J_{6}-9 J_{2} J_{4}-20 J_{3}^{2}+3 J_{2}^{3} \neq 0
$$

as rational expressions of $J_{2}, \ldots, J_{7}$. More precisely, we have

$$
\sigma_{1}=-\frac{9\left(3 J_{7}-3 J_{2} J_{5}+3 J_{3} J_{4}-J_{2}^{2} J_{3}\right)}{2\left(6 J_{6}-9 J_{2} J_{4}-20 J_{3}^{2}+3 J_{2}^{3}\right)} .
$$

and

$$
\sigma_{2}=\frac{4}{7} \sigma_{1}^{2}-\frac{1}{14} J_{2}, \quad \sigma_{3}=\frac{1}{24} J_{3}+\frac{1}{7} \sigma_{1}^{3}-\frac{1}{56} \sigma_{1} J_{2},
$$

Then $\lambda_{1}, \lambda_{2}, \lambda_{3}$ are recovered (up to their monodromy group) as the roots of the third degree polynomial in $\lambda$

$$
p(\lambda)=\lambda^{3}-\sigma_{1} \lambda^{2}+\sigma_{2} \lambda-\sigma_{3}
$$

But, to ensure that given $J_{2}, \ldots, J_{7} \in \mathbb{R}$ we can find $\lambda_{1}, \lambda_{2}, \lambda_{3} \in \mathbb{R}$, the following inequalities have to be satisfied

$$
\Delta_{2}=2 \sigma_{1}^{2}-6 \sigma_{2} \geq 0 \quad, \quad \Delta_{3}=\sigma_{1}^{2} \sigma_{2}^{2}-4 \sigma_{1}^{3} \sigma_{3}+18 \sigma_{1} \sigma_{2} \sigma_{3}^{2}-27 \sigma_{3}^{2} \geq 0
$$

The discriminant $\Delta_{3}$ and $\Delta_{2}$ can be rewritten, using $J_{2}, \ldots, J_{7}$, as

$$
\Delta_{2}=\frac{N_{2}}{14\left(6 J_{6}-9 J_{2} J_{4}-20 J_{3}{ }^{2}+3 J_{2}{ }^{3}\right)^{2}} \quad ; \quad \Delta_{3}=\frac{6 J_{6}-9 J_{2} J_{4}-20 J_{3}{ }^{2}+3 J_{2}{ }^{3}}{432}
$$


where

$$
\begin{aligned}
N_{2}= & 3645 J_{7}^{2}+7290 J_{2} J_{5} J_{7}-7290 J_{3} J_{4} J_{7}+2430 J_{2}{ }^{2} J_{3} J_{7}-36 J_{2} J_{6}{ }^{2} \\
& +108 J_{2}{ }^{2} J_{4} J_{6}+240 J_{2} J_{3}{ }^{2} J_{6}-36 J_{2}{ }^{4} J_{6}-3645 J_{2}{ }^{2} J_{5}{ }^{2}+7290 J_{2} J_{3} J_{4} J_{5} \\
& -2430 J_{2}{ }^{3} J_{3} J_{5}-3645 J_{3}{ }^{2} J_{4}{ }^{2}-81 J_{2}{ }^{3} J_{4}{ }^{2}+2070 J_{2}{ }^{2} J_{3}{ }^{2} J_{4} \\
& +54 J_{2}{ }^{5} J_{4}-400 J_{2} J_{3}{ }^{4}-285 J_{2}{ }^{4} J_{3}{ }^{2}-9 J_{2}{ }^{7} .
\end{aligned}
$$

We will summarize these results in the following proposition.

Proposition 5.12. A harmonic tensor $D \in \mathbb{H}^{4}$ is in the closed stratum $\bar{\Sigma}_{\left[\mathbb{D}_{2}\right]}$ if and only if the invariants $J_{2}(D), \cdots, J_{10}(D)$ satisfy the polynomial relations $(27)$, completed by the three equations

$$
S_{k}\left(J_{2}, \ldots J_{10}\right)=0, \quad k=8,9,10
$$

obtained from $J_{k}-p_{k}\left(\sigma_{1}, \sigma_{2}, \sigma_{3}\right)$, where we have substituted the corresponding rational expressions for the $\sigma_{i}$, and we have cleared the denominators. The inequalities

$$
6 J_{6}-9 J_{2} J_{4}-20 J_{3}^{2}+3 J_{2}^{3} \geq 0
$$

as well as

$$
\begin{aligned}
& 3645 J_{7}^{2}+7290 J_{2} J_{5} J_{7}-7290 J_{3} J_{4} J_{7}+2430 J_{2}{ }^{2} J_{3} J_{7}-36 J_{2} J_{6}{ }^{2}+108 J_{2}{ }^{2} J_{4} J_{6} \\
& +240 J_{2} J_{3}{ }^{2} J_{6}-36 J_{2}{ }^{4} J_{6}-3645 J_{2}{ }^{2} J_{5}{ }^{2}+7290 J_{2} J_{3} J_{4} J_{5}-2430 J_{2}{ }^{3} J_{3} J_{5} \\
& -3645 J_{3}{ }^{2} J_{4}{ }^{2}-81 J_{2}{ }^{3} J_{4}{ }^{2}+2070 J_{2}{ }^{2} J_{3}{ }^{2} J_{4}+54 J_{2}{ }^{5} J_{4} \\
& -400 J_{2} J_{3}{ }^{4}-285 J_{2}{ }^{4} J_{3}{ }^{2}-9 J_{2}{ }^{7} \geq 0
\end{aligned}
$$

are also required. $D$ is in the orthotropic class $\left[\mathbb{D}_{2}\right]$ if moreover inequality (29) is strict. In that case, it admits the normal form (26) where $\lambda_{1}, \lambda_{2}, \lambda_{3}$ are the roots of the third degree polynomial

$$
p(\lambda)=\lambda^{3}-\sigma_{1} \lambda^{2}+\sigma_{2} \lambda-\sigma_{3}
$$

Remark 5.13. Even if it is the core of the method, it is worth noting that, instead of $\lambda_{1}, \lambda_{2}, \lambda_{3}$, the three numbers $\sigma_{1}, \sigma_{2}, \sigma_{3}$ are uniquely defined on the open stratum: they are invariants of $D$ and can be written as rational expressions of $\left(J_{2}, \ldots, J_{7}\right)$. It should be noticed that it is also possible to express $\sigma_{1}, \sigma_{2}, \sigma_{3}$ as rational functions of only $\left(J_{2}, \ldots, J_{5}\right)$ on an open subset of $\Sigma_{\left[\mathbb{D}_{2}\right]}$. We will, however, not give these formulas here.

Remark 5.14. All second rank tensors in $\mathbb{H}^{2}$ covariant to $D$ commute. 


\subsection{Bifurcation conditions for tensors in $\mathbb{H}^{4}$}

An important observation has to be made. Mathematical results [32] tell us that the invariant algebra of $\mathbb{H}^{4}$ is generated by the 9 fundamental invariants $\left(J_{2}, \ldots, J_{10}\right)$. In this set 6 invariants $\left(J_{2}, \ldots, J_{7}\right)$ are algebraically independent, meanwhile the others are linked to the formers by polynomial relations. As discussed before, it is a wellknown fact that this algebra separates the orbits. But, as demonstrated exhaustively in this section, for any class with a finite monodromy group ${ }^{13}$, the first 6 invariants $\left(J_{2}, \ldots, J_{7}\right)$ are necessary and sufficient to separate the orbits inside each of these classes. This observation can be summed-up by the following theorem.

Theorem 5.15. 1. For the cubic, the transversely isotropic, the trigonal and the tetragonal classes in $\mathbb{H}^{4}\left(\mathbb{R}^{3}\right)$, the first four invariants $\left(J_{2}, J_{3}, J_{4}, J_{5}\right)$ separate the orbits inside the class.

2. For the orthotropic class, the first six invariants $\left(J_{2}, J_{3}, J_{4}, J_{5}, J_{6}, J_{7}\right)$ separate the orbits inside the class.

We will now give bifurcation conditions on the $J_{k}$ which make explicit how we "travel" from a given isotropy class to another. More precisely, so far, we have given necessary and sufficient conditions to belong to a closed stratum $\bar{\Sigma}_{[H]}$ with finite monodromy group. A closed $\bar{\Sigma}_{[H]}$ is defined by a finite set of polynomial relations and inequalities on the $J_{k}$. Given two adjacent strata $\bar{\Sigma}_{\left[H_{1}\right]} \subset \bar{\Sigma}_{\left[H_{2}\right]}$, the bifurcation conditions, detailed on figure 2 , correspond to the relations which need to be added for a tensor in $\bar{\Sigma}_{\left[H_{2}\right]}$ to belong to $\bar{\Sigma}_{\left[H_{1}\right]}$.

\section{Relation with the characteristic polynomial}

In this section, we will investigate the relations between the invariants of $\mathbb{H}^{4}$, the coefficients of the characteristic polynomial of $\underline{D}$ and Betten's invariants [7].

Proposition 6.1. Let $\underline{D} \in \mathbb{H}^{4}$. The coefficients of the characteristic polynomial of $\underline{D}$ are $\mathrm{SO}(3)$-invariants of $D$. They can be expressed in terms of the invariants $J_{2}, \ldots, J_{5}$ defined above. We have

$$
\chi_{D}(z)=z^{6}-\frac{1}{2} J_{2} z^{4}-\frac{1}{3} J_{3} z^{3}+\frac{1}{5}\left(J_{2}^{2}-2 J_{4}\right) z^{2}+\frac{2}{25}\left(J_{2} J_{3}-3 J_{5}\right) z
$$

Proof. Notice first that $z=0$ is always an eigenvalue of $\underline{D}$. Indeed, if $\mathbf{q}$ stands for the standard metric on $\mathbb{R}^{3}$, we have $\underline{D} \mathbf{q}=\operatorname{tr}_{12}(D)=0$. The coefficient of $z^{k}$ in $\chi_{D}$ is a homogeneous invariant polynomial of degree $6-k$ for $1 \leq k \leq 4$. Therefore, it can be expressed as a polynomial in $J_{2}(D), \cdots, J_{5}(D)$. The computation of this last expression reduces therefore to identify some real coefficients. This can be done by specializing on particular values or on some particular strata where it is easy to compute, the cubic or the transverse isotropic stratum, for instance.

13 i.e., for all symmetry classes, except the monoclinic and the triclinic classes 


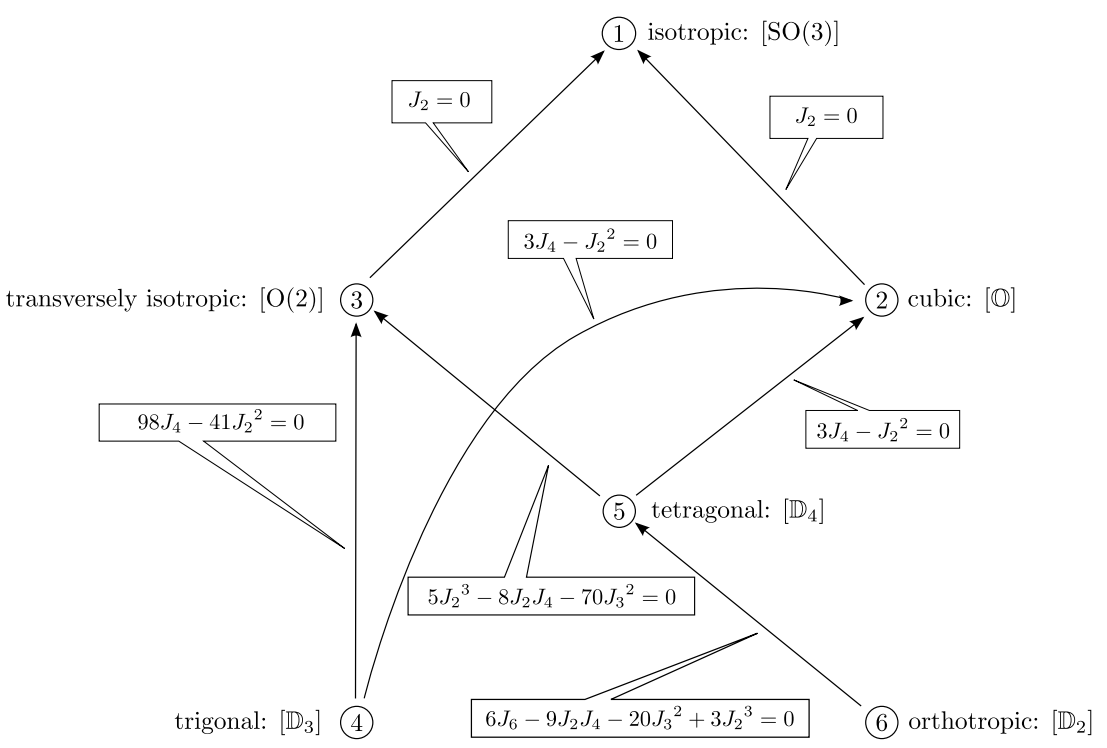

Fig. 2 Bifurcation paths for finite monodromy isotropy classes in $\mathbb{H}^{4}$.

Besides the characteristic polynomial, Betten [7] has introduced a two variables, invariant polynomial of the elasticity tensor. It is defined as

$$
\mathscr{B}_{C}(\lambda, \mu)=\operatorname{det}\left(\underline{\mathbf{C}}-\underline{\mathbf{C}}_{\lambda, \mu}\right),
$$

where

$$
\underline{\mathbf{C}}_{\lambda, \mu}=\left(\begin{array}{cccccc}
\lambda+2 \mu & \lambda & \lambda & 0 & 0 & 0 \\
\lambda & \lambda+2 \mu & \lambda & 0 & 0 & 0 \\
\lambda & \lambda & \lambda+2 \mu & 0 & 0 & 0 \\
0 & 0 & 0 & 2 \mu & 0 & 0 \\
0 & 0 & 0 & 0 & 2 \mu & 0 \\
0 & 0 & 0 & 0 & 0 & 2 \mu
\end{array}\right)
$$

is the totally isotropic tensor. Notice that $\mathscr{B}_{D}(0, \mu)=\chi_{D}(2 \mu)$ and that $\mathscr{B}_{C}$ is of degree lower than 1 in $\lambda$ (indeed, this can be observed by subtracting the first line to the second line and to the third line in the determinant). The interset of this polynomial is that it may generate other invariants than the ones obtained from the characteristic polynomial. However, it does not bring any new invariants for a tensor $D \in \mathbb{H}^{4}\left(\mathbb{R}^{3}\right)$. Indeed, we have the following result.

Lemma 6.2. The Betten polynomial of a harmonic tensor $D \in \mathbb{H}^{4}\left(\mathbb{R}^{3}\right)$ is related to its characteristic polynomial by the formula

$$
\mathscr{B}_{D}(\lambda, \mu)=(3 \lambda+2 \mu) \chi_{D}^{r}(2 \mu)
$$

where $\chi_{D}(\lambda)=\lambda \chi_{D}^{r}(\lambda)$. 
Proof. For a harmonic tensor $D=\left(D_{i j k l}\right)$ with matrix representation $\underline{D}=\left(d_{m n}\right)$ (see remark 2.1), we have

$$
\left(\operatorname{tr}_{12} D\right)_{i j}:=\sum_{k=1}^{3} D_{k k i j}=0, \quad 1 \leq i, j \leq 3,
$$

and therefore

$$
\sum_{m=1}^{3} d_{m n}=0, \quad 1 \leq n \leq 6 .
$$

Thus, if $L_{m}(1 \leq m \leq 6)$ are the lines of the determinant $\operatorname{det}\left(\underline{D}-\underline{\mathbf{C}}_{\lambda, \mu}\right)$, the substitution $L_{1} \rightarrow L_{1}+L_{2}+L_{3}$ shows that there is a factorisation

$$
\mathscr{B}_{D}(\lambda, \mu)=(3 \lambda+2 \mu) P(\lambda, \mu) \text {. }
$$

But $\mathscr{B}_{D}(\lambda, \mu)$ is of degree lower than 1 in $\lambda$ and hence $P(\lambda, \mu)=P(\mu)$ does not depend on $\lambda$. Now, for $\lambda=0$ we get

$$
\mathscr{B}_{D}(0, \mu)=\chi_{D}(2 \mu)=(2 \mu) P(\mu),
$$

from which we deduce that $P(\mu)=\chi_{D}^{r}(2 \mu)$. This achieves the proof.

Corollary 6.3. The invariants defined by the coefficients of the Betten polynomial do not separate the orbits of $\mathbb{E}$ la.

Proof. If this was the case, it would also separate the orbits of $\mathbb{H}^{4}$ but then lemma 6.2 and proposition 6.1 would imply that $J_{2}, J_{3}, J_{4}, J_{5}$ separate the orbits of $\mathbb{H}^{4}$, which is false.

\section{Conclusion}

In this paper, five sets of algebraic relations were provided to identify the following symmetry classes in $\mathbb{H}^{4}$ (section 5$)$ : orthotropic $\left(\left[\mathbb{D}_{2}\right]\right)$, trigonal $\left(\left[\mathbb{D}_{3}\right]\right)$, tetragonal $\left(\left[\mathbb{D}_{4}\right]\right)$, transverse isotropic $([\mathrm{SO}(2)])$ and cubic $([\mathbb{O}])$. These relations are invariant necessary conditions for an elasticity tensor to belong to one of these classes. We consider this paper as a first step towards a more systematic use of invariant-based methods in continuum mechanics. Based on the geometrical framework introduced in this paper, the following extensions of the current method can be considered:

- extend the current polynomial-characterization of isotropy classes to ones having continuous monodromy group,

- identify the transformation matrix that bring a tensor to its normal form,

- tackle the increasing degree of the algebraic relations for low symmetry groups. It might be interesting to characterize classes in terms of second-order covariants.

Such an approach was conducted here in the context of classical elasticity, but the framework used allows a direct extension of this method to other kinds of anisotropic tensorial behaviours. It can be extended to the study of anisotropic features of piezoelasticity, flexoelasticity or strain-gradient elasticity. Furthermore, from a more practical viewpoint, we aim at testing our approach on experimental situations. This would allow to compare the proposed approach with the more classical ones. 


\section{A Appendix: Closed subgroups of $\mathrm{SO}(3)$ and their normalizers}

Every closed subgroup of $\mathrm{SO}(3)$ is conjugate to one of the following list [19]

$$
\mathrm{SO}(3), \mathrm{O}(2), \mathrm{SO}(2), \mathbb{D}_{n}(n \geq 2), \mathbb{Z}_{n}(n \geq 2), \mathbb{T}, \mathbb{O}, \mathbb{I}, \text { and } \mathbb{1}
$$

where:

- $\mathrm{O}(2)$ is the subgroup generated by all the rotations around the $z$-axis and the order 2 rotation $\sigma$ : $(x, y, z) \mapsto(x,-y,-z)$ around the $x$-axis.

- $\mathrm{SO}(2)$ is the subgroup of all the rotations around the $z$-axis.

- $\mathbb{Z}_{n}$ is the unique cyclic subgroup of order $n$ of $\mathrm{SO}(2)$, the subgroup of rotations around the $z$-axis.

- $\mathbb{D}_{n}$ is the dihedral group. It is generated by $\mathbb{Z}_{n}$ and $\sigma:(x, y, z) \mapsto(x,-y,-z)$.

- $\mathbb{T}$ is the tetrahedral group, the (orientation-preserving) symmetry group of a tetrahedron. It has order 12.

- $\mathbb{O}$ is the octahedral group, the (orientation-preserving) symmetry group of a cube or octahedron. It has order 24.

- $\mathbb{I}$ is the icosahedral group, the (orientation-preserving) symmetry group of a icosahedra or dodecahedron. It has order 60.

- $\mathbb{1}$ is the trivial subgroup, containing only the unit element.

The poset of conjugacy classes of closed subgroups of $\mathrm{SO}(3)$ is completely described [19] by the following inclusion of subgroups (which generates order relations between conjugacy classes)

$$
\begin{aligned}
& \mathbb{Z}_{n} \subset \mathbb{D}_{n} \subset \mathrm{O}(2) \quad(n \geq 2), \\
& \mathbb{Z}_{n} \subset \mathbb{Z}_{m} \text { and } \mathbb{D}_{n} \subset \mathbb{D}_{m}, \quad(\text { if } n \text { divides } m), \\
& \mathbb{Z}_{2} \subset \mathbb{D}_{n} \quad(n \geq 2), \\
& \mathbb{Z}_{n} \subset \mathrm{SO}(2) \subset \mathrm{O}(2) \quad(n \geq 2),
\end{aligned}
$$

and the arrows in figure 3 which complete the poset, taking account of the exceptional subgroups $\mathbb{O}, \mathbb{T}, \mathbb{I}$ (beware that figure 3 cannot be realized by an inclusion diagram between representatives of these conjugacy classes).

Remark A.1. Notice that $\mathbb{O}$ and $\mathbb{I}$ are maximal subgroups of $\mathrm{SO}(3)$ which both contained $\mathbb{T}$.

Recall that the normalizer of a subgroup $H$ of a group $G$, denoted by $N(H)$ is the biggest subgroup of $G$ in which $H$ is normal. It is defined by

$$
N(H):=\left\{g \in G ; g H g^{-1}=H\right\} .
$$

Proposition A.2. The normalizers of the subgroups in the list (32) are given below.

$$
\begin{aligned}
& N(\mathrm{SO}(3))=\mathrm{SO}(3), \quad N(\mathrm{O}(2))=\mathrm{O}(2), \\
& N(\mathbb{O})=\mathbb{O}, \quad N(\mathbb{I})=\mathbb{I}, \quad N(\mathbb{T})=\mathbb{O}, \\
& N(\mathbb{1})=\mathrm{SO}(3), \quad N\left(\mathbb{Z}_{n}\right)=\mathrm{O}(2) \text { for } n \geq 2, \quad N\left(\mathbb{D}_{n}\right)=\mathbb{D}_{2 n} \text { for } n \geq 3,
\end{aligned}
$$

and $N\left(\mathbb{D}_{2}\right)=\mathbb{O}$. Moreover, the quotient groups are given by

$$
\begin{aligned}
& N(\mathrm{SO}(3)) / \mathrm{SO}(3)=\mathbb{1}, \quad N(\mathrm{O}(2)) / \mathrm{O}(2)=\mathbb{1}, \\
& N(\mathbb{O}) / \mathbb{O}=\mathbb{1}, \quad N(\mathbb{I}) / \mathbb{I}=\mathbb{1}, \\
& N(\mathrm{SO}(2)) / \mathrm{SO}(2)=\mathbb{Z}_{2}, \\
& N(\mathbb{1}) / \mathbb{1}=\mathrm{SO}(3), \\
& N\left(\mathbb{Z}_{n}\right) / \mathbb{Z}_{n}=\mathrm{O}(2) \text { for } n \geq 2, \\
& N(\mathbb{T}) / \mathbb{T}=\mathbb{Z}_{2}, \\
& N\left(\mathbb{D}_{n}\right) / \mathbb{D}_{n}=\mathbb{Z}_{2} \text { for } n \geq 3,
\end{aligned}
$$

and $N\left(\mathbb{D}_{2}\right) / \mathbb{D}_{2}=\mathfrak{S}_{3}$, the symmetric group of 3 elements.

Acknowledgements This work was supported by the CNRS interdisciplinary project PEPS Maths-ST2I 09-99. It is a pleasure to thank Christian Miebach, for his careful reading of the first manuscript of this paper and for helpful remarks. 


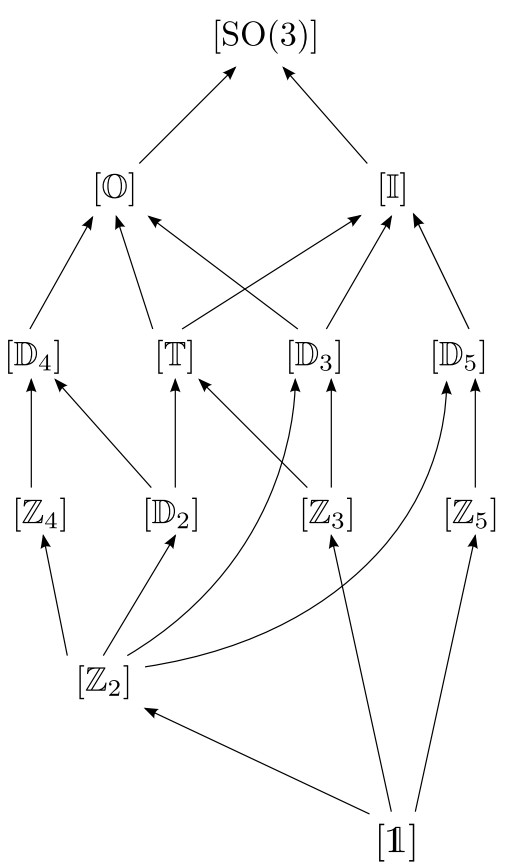

Fig. 3 Exceptional classes in the poset of conjugacy classes of closed subgroup of $\mathrm{SO}(3)$.

\section{References}

1. Abud, M., Sartori, G.: The geometry of orbit-space and natural minima of Higgs potentials. Phys. Lett. B 104(2), 147-152 (1981)

2. Abud, M., Sartori, G.: The geometry of spontaneous symmetry breaking. Ann. Physics 150(2), 307$372(1983)$

3. Auffray, N.: Démonstration du théorème de Hermann à partir de la méthode Forte-Vianello. C.R Mecanique 336(5), 458-463 (2008)

4. Auffray, N.: Analytical expressions for anisotropic tensor dimension. C.R. Mecanique 338(5), 260265 (2010)

5. Backus, G.: A geometrical picture of anisotropic elastic tensors. Rev. Geophys. 8(3), 633-671 (1970)

6. Baerheim, R.: Harmonic decomposition of the anisotropic elasticity tensor. Q. J. Mech. Appl. Math. 46(3), 391-418 (1993)

7. Betten, J.: Irreducible invariants of fourth-order tensors. Math. Modelling 8, 29-33 (1987)

8. Boehler, J.P., Kirillov Jr., A.A., Onat, E.T.: On the polynomial invariants of the elasticity tensor. J. Elasticity 34(2), 97-110 (1994)

9. Bóna, A., Bucataru, I., Slawinski, M.: Characterization of Elasticity-Tensor Symmetries Using SU(2). J. Elasticity 75(3), 267-289 (2004)

10. Bóna, A., Bucataru, I., Slawinski, M.: Coordinate-free Characterization of the Symmetry Classes of Elasticity Tensors. J. Elasticity 87(2), 109-132 (2007)

11. Bóna, A., Bucataru, I., Slawinski, M.: Space of $S O(3)$-orbits of elasticity tensors. Arch. Mech. 60(2), 123-138 (2008)

12. Bredon, G.E.: Introduction to compact transformation groups. Academic Press, New York (1972). Pure and Applied Mathematics, Vol. 46

13. Coste, M.: An Introduction to Semialgebraic Geometry (2002). Université de Rennes

14. Cowin, S.C.: Properties of the anisotropic elasticity tensor. Q. J. Mech. Appl. Math. 42, 249-266 (1989)

15. Cowin, S.C., Mehrabadi, M.M.: Eigentensors of linear anisotropic elastic materials. Q. J. Mech. Appl. Math. 43, 15-41 (1990) 
16. Cox, D., Little, J., O’Shea, D.: Ideals, varieties, and algorithms, third edn. Undergraduate Texts in Mathematics. Springer, New York (2007)

17. Forte, S., Vianello, M.: Symmetry classes for elasticity tensors. J. Elasticity 43(2), 81-108 (1996)

18. Francois, M., Geymonat, G., Berthaud, Y.: Determination of the symmetries of an experimentally determined stiffness tensor: application to acoustic measurements 35(31), 4091-4106 (1998)

19. Golubitsky, M., Stewart, I., Schaeffer, D.G.: Singularities and groups in bifurcation theory. Vol. II, Applied Mathematical Sciences, vol. 69. Springer-Verlag, New York (1988)

20. Herman, B.: Some theorems of the theory of anisotropic media 48(2), 89-92 (1945)

21. Hilbert, D.: Theory of algebraic invariants. Cambridge University Press, Cambridge (1993)

22. Norris, A.N.: On the acoustic determination of the elastic moduli of anisotropic solids and acoustic conditions for the existence of symmetry planes. Quart. J. Mech. Appl. Math. 42, 413-426 (1989)

23. Olive, M., Auffray, N.: Symmetry classes for even-order tensors (2013)

24. Olver, P.J.: Group theoretic classification of conservation laws in elasticity. In: Systems of nonlinear partial differential equations (Oxford, 1982), NATO Adv. Sci. Inst. Ser. C Math. Phys. Sci., vol. 111, pp. 323-331. Reidel, Dordrecht (1983)

25. Olver, P.J.: Canonical anisotropic elastic moduli. In: Modern theory of anisotropic elasticity and applications (Research Triangle Park, NC, 1990), pp. 325-339. SIAM, Philadelphia, PA (1991)

26. Olver, P.J.: Classical invariant theory, London Mathematical Society Student Texts, vol. 44. Cambridge University Press, Cambridge (1999)

27. Procesi, C., Schwarz, G.: Inequalities defining orbit spaces. Invent. Math. 81(3), 539-554 (1985)

28. Procesi, C., Schwarz, G.W.: The geometry of orbit spaces and gauge symmetry breaking in supersymmetric gauge theories. Phys. Lett. B 161(1-3), 117-121 (1985)

29. Rychlewski, J.: On hooke's law. J. Appl. Math. Mech. 48(3), 303-314 (1984)

30. Sartori, G., Valente, G.: Tools in the orbit space approach to the study of invariant functions: rational parametrization of strata. J. Phys. A 36(7), 1913-1929 (2003)

31. Schwarz, G.W.: Lifting smooth homotopies of orbit spaces. Inst. Hautes Études Sci. Publ. Math. (51), 37-135 (1980)

32. Shioda, T.: On the graded ring of invariants of binary octavics. Amer. J. Math. 89, 1022-1046 (1967)

33. Thurston, W.P.: Three-dimensional geometry and topology. Vol. 1, Princeton Mathematical Series, vol. 35. Princeton University Press, Princeton, NJ (1997) 